\title{
BAYESIAN COMPRESSIVE SENSING FOR APPROXIMATELY SPARSE SIGNALS AND APPLICATION TO STRUCTURAL HEALTH MONITORING SIGNALS FOR DATA LOSS RECOVERY
}

\author{
Yong Huang ${ }^{1,2}$, James L. Beck ${ }^{2 *}$, Stephen $\mathrm{Wu}^{3}$, Hui $\mathrm{Li}^{1}$ \\ ${ }^{1}$ Key Lab of Structural Dynamic Behavior and Control of the Ministry of Education, \\ Harbin Institute of Technology, Harbin 150090, China \\ ${ }^{2}$ Division of Engineering and Applied Science, California Institute of Technology, Pasadena, CA 91125, USA \\ ${ }^{3}$ Institute of Statistical Mathematics, Tokyo 190-8562, Japan
}

\begin{abstract}
The theory and application of compressive sensing (CS) have received a lot of interest in recent years. The basic idea in CS is to use a specially-designed sensor to sample signals that are sparse in some basis (e.g. wavelet basis) directly in a compressed form, and then to reconstruct (decompress) these signals accurately using some inversion algorithm after transmission to a central processing unit. However, many signals in reality are only approximately sparse, where only a relatively small number of the signal coefficients in some basis are significant and the remaining basis coefficients are relatively small but they are not all zero. In this case, perfect reconstruction from compressed measurements is not expected. In this paper, a Bayesian CS algorithm is proposed for the first time to reconstruct approximately sparse signals. A robust treatment of the uncertain parameters is explored, including integration over the prediction-error precision parameter to remove it as a "nuisance" parameter, and introduction of a successive relaxation procedure for the required optimization of the basis coefficient hyper-parameters. The performance of the algorithm is investigated using compressed data from synthetic signals and real signals from structural health monitoring systems installed on a space-frame structure and on a cable-stayed bridge. Compared with other state-of-the-art CS methods, including our previously-published Bayesian method, the new CS algorithm shows superior performance in reconstruction robustness and posterior uncertainty quantification, for approximately sparse signals. Furthermore, our method can be utilized for recovery of lost data during wireless transmission, even if the level of sparseness in the signal is low.

*Corresponding author.

E-mail address: jimbeck@ caltech.edu (James L. Beck)
\end{abstract}




\section{Keywords}

Bayesian compressive sensing, compressed sensing, approximately sparse signals, data loss recovery, wireless sensor networks, structural health monitoring

\section{INTRODUCTION}

In the last decade, a new type of sampling theory named compressive (or compressed) sensing has become a very active research topic and shows promise for many applications [1-3]. In a CS sensor, the signal is digitized and linearly projected onto a lower-dimensional space by multiplying with a rectangular matrix. It has been found that a sufficiently sparse signal can be reconstructed (decompressed) with high accuracy from a compressed version by using a least-squares method with $l_{1}$-norm regularization [1,4], even though the amount of compressed sampling measurements is insufficient by the Nyquist-Shannon criterion. The data reconstruction takes advantage of the signal's sparseness in terms of some basis, allowing only solutions with a small number of nonzero basis coefficients. This new technique presents an efficient signal processing paradigm by merging traditional signal sensing and compression into a single phase, therefore increasing the efficiency of data transfer and storage.

In the past few years, many CS reconstruction methods have been proposed to solve the CS reconstruction problem using $l_{1}$-norm minimization or its extensions (e.g., [1,4-7]). Alternative methods are $l_{0}$-norm minimization, such as iterative hard-thresholding methods [8-9], and non-convex $l_{p}$-pseudo-norm $(0<p<1)$ minimization such as iteratively re-weighted least squares [10-11]. However, all these methods are based on deterministic frameworks that provide only a point estimate of the basis coefficients to specify the signal reconstruction, so the reconstruction uncertainties are not explicitly quantified. These uncertainties are important, however, in order to give a measure of confidence in the reconstruction of the original signal. In addition, uncertainty quantification allows optimal design of the CS measurements based on the goal of reducing the reconstruction uncertainty.

Recently, Bayesian Compressive Sensing (BCS) has been proposed [12-13]. The Bayesian CS reconstruction algorithm utilizes the sparse Bayesian learning method [14-17] to infer posterior probability distributions over the basis coefficients that suppress the basis terms that do not contribute significantly to the reconstructed signals. The effective dimensionality (number of nonzero signal coefficients) of the signal reconstruction model is determined automatically as part of the full Bayesian inference procedure, and so it does 
not require the tuning of the signal sparseness or noise levels that is required in the traditional CS methods. Furthermore, many compressive sensing recovery schemes can be interpreted using a Bayesian point of view. For example, least-squares with $l_{1}$-norm regularization can be formulated as maximum a posteriori (MAP) estimation under a Bayesian model with a sparseness-inducing Laplace prior distribution [13].

Recently, we found that current BCS reconstruction algorithms suffer from a robustness problem: there are local maxima that may trap the hyperparameter optimization algorithm when the number of measurements $K$ becomes much smaller than the number of signal degrees of freedom $N$, leading to non-robust signal reconstruction [18]. We then presented improvements to the BCS reconstruction method to enhance its robustness so that even higher compression ratios $N / K$ could be used. However, all of these BCS reconstruction algorithms are predicated on the prior assumption that the original signal is very sparse in some basis, i.e., only relatively few basis components have nonzero magnitudes, with all remaining components equal to zero. In addition, posterior uncertainty quantification for these methods is unreliable, which may lead to over confidence in the signal reconstruction because the uncertainties of the basis coefficient hyper-parameters are not explicitly treated.

For real applications of compressive sensing, the signals are usually only approximately sparse, where their main energy (in terms of the sum of the squares of the basis coefficients) is concentrated in only a few basis components and the other components are relatively small but they are not all zero. For example, civil infrastructure systems are usually subjected to wide-band transient ambient excitation, and structural monitoring signals of interest are also corrupted by a combination of measurement noise and unknown environmental excitation. The strict sparseness level (the total number of zero basis components) is therefore low and so highly accurate reconstruction is unlikely to be achieved with limited measurements. CS reconstruction of approximately sparse signals has attracted recent theoretical interest [19-21]. However, to our knowledge, no Bayesian method for reconstructing approximately sparse signals has been presented.

In recent years, CS techniques have been applied to structural monitoring. One motivation is to reduce the power demand for wireless transmission form each sensor in a structural network [18,22,23]. Another application is the work of Bao et al. [24] to recover missing data during wireless transmission [25], which can be a problem in practice, since the partial loss of CS compressed measurements can be regarded as higher data compression in the CS framework. Other applications in structural monitoring are to modal identification [26] and to damage detection $[27,28]$. In this work, we apply our BCS method for robust reconstruction of approximately sparse 
signals from two real structural monitoring systems, and also for recovery of lost data during wireless transmission in a monitoring system.

For quantification of the reconstruction uncertainties when using compressed data from approximately sparse signals, we favor a hierarchical Bayesian approach based on sparse Bayesian learning (SBL) [14-17]. We present two BCS algorithms for signal reconstruction in Section 2: the first one, BCS-MPE (Bayesian Compressive Sensing using MAP estimation of the uncertain Prediction-Error precision) is a variant of the method proposed in [18], and the second one, BCS-IPE (Bayesian Compressive Sensing using Integration over the uncertain Prediction-Error precision) is a further enhancement of the robustness in reconstruction of approximately sparse signals. The two algorithms are explicitly compared in terms of their signal reconstruction robustness. From the presented results with synthetic and real signals in Section 3, BCS-IPE algorithm is found to be superior in this regard and in its ability to recover a signal from partial data loss during wireless transmission.

\section{BAYESIAN COMPRESSIVE SENSING AND PROPOSED ALGORITHMS}

We consider an unknown signal $\overline{\mathbf{x}}$ with $N$ degrees of freedom: $\overline{\mathbf{x}}=[\bar{x}(1), \cdots \bar{x}(N)]^{T}$ in $\mathbb{R}^{N}$, which is represented by a set of orthonormal basis vectors as

$$
\overline{\mathbf{x}}=\boldsymbol{\Psi} \overline{\mathbf{w}}_{a s}=\boldsymbol{\Psi}\left(\overline{\mathbf{w}}_{s}+\overline{\mathbf{w}}_{e}\right)
$$

where $\boldsymbol{\Psi}=\left\lceil\boldsymbol{\Psi}_{1}, \cdots, \boldsymbol{\Psi}_{N}\right\rceil$ is the $N \times N$ unitary matrix with the basis of $N \times 1$ vectors $\left\{\boldsymbol{\Psi}_{n}\right\}_{n=1}^{N}$ as columns. The unknown vector $\overline{\mathbf{w}}_{a s}=\boldsymbol{\Psi}^{T} \overline{\mathbf{x}}$ of basis coefficients is assumed to be approximately sparse where $T$ of its components are nonzero and we call $(N-T)$ the strict sparseness level of the signal $\overline{\mathbf{x}}$. We define $\overline{\mathbf{w}}_{S}$ to represent an $N$-dimensional $T_{s}$-sparse vector that is identical to the vector $\overline{\mathbf{w}}_{a s}$ for the $T_{s}$ basis components with larger magnitudes while all other components are set to zero. The difference between the two vectors $\overline{\mathbf{w}}_{a s}-\overline{\mathbf{w}}_{s}$ is denoted by $\overline{\mathbf{w}}_{e}$, which has exactly $\left(T-T_{s}\right)$ non-zero components that are the smaller magnitude components in $\overline{\mathbf{w}}_{a s}$. The total number $\left(N-T_{s}\right)$ of the zero components of $\overline{\mathbf{w}}_{s}$ represents the effective sparseness level of the signal $\overline{\mathbf{x}}$ with respect to the basis $\left\{\boldsymbol{\Psi}_{n}\right\}$.

In the CS framework, one infers the signal coefficients vector $\mathbf{w}$ of interest from compressed data instead of directly sampling the actual signal $\overline{\mathbf{x}}$. Let $\mathbf{y}$ in $\mathbb{R}^{K}$ represent the compressed measurement from $K \ll N$ linear projections of the original signal $\overline{\mathbf{x}}$ using a chosen $K \times N$ random projection matrix $\boldsymbol{\Phi}$ that is built into 
the sensor (typically, each element in $\boldsymbol{\Phi}$ is drawn from zero-mean Gaussian distribution $\mathcal{N}(0,1)$ ):

$$
\mathbf{y}=\boldsymbol{\Phi} \overline{\mathbf{x}}+\mathbf{r}
$$

where $\mathbf{r}$ represents any measurement noise, that is, errors from sensing and projection of $\overline{\mathbf{x}}$, which will be relatively small for modern sensors. Incorporating (1), we can rewrite $\mathbf{y}$ as

$$
\mathbf{y}=\boldsymbol{\Theta} \overline{\mathbf{w}}_{a s}+\mathbf{r}=\boldsymbol{\Theta} \overline{\mathbf{w}}_{s}+\Theta \overline{\mathbf{w}}_{e}+\mathbf{r}
$$

where $\boldsymbol{\Theta}=\boldsymbol{\Phi} \boldsymbol{\Psi}=\left\lceil\boldsymbol{\Theta}_{1}, \cdots, \boldsymbol{\Theta}_{N}\right\rceil$.

For signal reconstruction, the compressed data $\mathbf{y}$ is represented as:

$$
\mathbf{y}=\boldsymbol{\Theta} \mathbf{w}+\boldsymbol{\Theta}\left(\overline{\mathbf{w}}_{a s}-\mathbf{w}\right)+\mathbf{r}=\boldsymbol{\Theta} \mathbf{w}+\mathbf{e}
$$

where $\mathbf{e}=\boldsymbol{\Theta}\left(\overline{\mathbf{w}}_{a s}-\mathbf{w}\right)+\mathbf{r}$ represents the unknown prediction error in $\mathbf{y}$ when the unknown signal is modeled by $\mathbf{x}=\boldsymbol{\Psi}_{\mathbf{w}}$, combined with any measurement noise $\mathbf{r}$. Because we want $\mathbf{w}$ to pick up the components of $\overline{\mathbf{w}}_{a s}$, especially the larger magnitude ones, we want $\mathbf{e}$ to be small. For data compression, we will have $K \ll N$, so (4) leads to an ill-posed inversion problem to find the sparse weights $\mathbf{w}$, and hence to find the signal $\mathbf{x}$ in $\mathbb{R}^{N}$, from data $\mathbf{y}$ in $\mathbb{R}^{K}$. In order to reduce the number of solutions for such an underdetermined system, one can impose an extra constraint of sparseness by allowing only solutions which have a small number of nonzero basis coefficients. A typical approach is to use an $l_{1}$-norm regularized least-squares formulation to estimate "optimal" basis coefficients [1,6]:

$$
\widetilde{\mathbf{w}}=\arg \min \left\{\|\mathbf{y}-\boldsymbol{\Theta} \mathbf{w}\|_{2}^{2}+\lambda\|\mathbf{w}\|_{1}\right\}
$$

where the penalty parameter $\lambda$ controls the trade-off between how well the data is fitted (first term) and how sparse the signal is (second term). In contrast, as a continuation of our work in [18], we use sparse Bayesian learning to infer the plausible values of $\mathbf{w}$ based on the compressed data $\mathbf{y}$, a procedure called Bayesian compressive sensing (BCS) [12].

\subsection{Bayesian modeling for compressive sensing reconstruction}

Following [18], we get a Gaussian likelihood function for parameters $\mathbf{w}$ and $\beta$ based on $\mathbf{y}$ :

$$
p(\mathbf{y} \mid \mathbf{w}, \beta)=\mathcal{N}\left(\mathbf{y} \mid \Theta \mathbf{w}, \beta^{-1} \mathbf{I}_{K}\right)=\left(2 \pi \beta^{-1}\right)^{-\frac{K}{2}} \exp \left(-\frac{\beta}{2}\|\mathbf{y}-\boldsymbol{\Theta} \mathbf{w}\|_{2}^{2}\right)
$$

where $\beta$ is the precision parameter for a Gaussian maximum-entropy probability model for the components of the uncertain prediction error $\mathbf{e}$ in (4). The logarithm of this likelihood corresponds to the first term of (5) in the deterministic CS data inversion. Following [18] again, a Gamma conjugate prior for the likelihood in (6) is taken for $\beta$ : 


$$
p\left(\beta \mid a_{0}, b_{0}\right)=\operatorname{Gamma}\left(\beta \mid a_{0}, b_{0}\right)=\frac{b_{0} a_{0}}{\Gamma\left(a_{0}\right)} \beta^{a_{0}-1} \exp \left(-b_{0} \beta\right)
$$

Often, the prior PDFs for $\mathrm{a}_{0}$ and $\mathrm{b}_{0}$ are taken as uniform over large open intervals that start at zero. However, in the present case, this leads to improper priors since we need $a_{0} \rightarrow \infty$ and $b_{0} \rightarrow \infty$. To take a proper prior, the Exponential priors are defined instead: $p\left(a_{0}\right)=\operatorname{Exp}\left(a_{0} \mid \tau\right)$ and $p\left(b_{0}\right)=\operatorname{Exp}\left(b_{0} \mid \tau\right)$, and we fix the rate parameter $\tau$ to very small values: e.g., $\tau=10^{-10}$. These priors are almost flat since the mean $\mathbf{E}\left(a_{0}\right)=$ $\mathbf{E}\left(b_{0}\right)=10^{10}$ and variance $\operatorname{Var}\left(a_{0}\right)=\operatorname{Var}\left(b_{0}\right)=10^{100}$.

In the sparse Bayesian learning formulation, sparseness is promoted by placing a special prior on the signal basis coefficients $\mathbf{w}$ known as the automatic relevance determination prior (ARD prior):

$$
p\left(\mathbf{w} \mid \boldsymbol{\Sigma}_{0}\right)=\prod_{n=1}^{N} \mathcal{N}\left(w_{n} \mid 0, \sigma_{n}^{2}\right)=\prod_{n=1}^{N}\left[\left(2 \pi \sigma_{n}^{2}\right)^{-1 / 2} \exp \left\{-\frac{1}{2} \sigma_{n}^{-2} w_{n}^{2}\right\}\right]
$$

where the prior covariance matrix $\boldsymbol{\Sigma}_{0}=\operatorname{diag}\left(\sigma_{1}^{2}, \ldots, \sigma_{N}^{2}\right)$ so that $\sigma_{n}^{2}$ is the prior variance for $\mathrm{w}_{n}$. Tipping [16] has shown that maximizing the model evidence (see below for its definition) with respect to all of the $\sigma_{n}^{2}$ controls the model sparseness because many $\sigma_{n}^{2} \rightarrow 0$, implying $w_{n} \rightarrow 0$, thereby having an effect similar to the regularization term in (5). In order to allow the tractability of an integration involved later, we incorporate the prediction error precision $\beta$ in the ARD prior by replacing each $\sigma_{n}^{2}$ by $\alpha_{n}=1 /\left(\beta \sigma_{n}^{2}\right)$ to get the following prior PDF (probability density function) conditional on the hyper-parameters $\alpha$ and $\beta$ :

$$
p(\mathbf{w} \mid \boldsymbol{\alpha}, \beta)=\prod_{n=1}^{N} \mathcal{N}\left(w_{n} \mid 0, \beta^{-1} \alpha_{n}^{-1}\right)=\prod_{n=1}^{N}\left[(2 \pi)^{-1 / 2}\left(\beta \alpha_{n}\right)^{1 / 2} \exp \left\{-\frac{1}{2} \beta \alpha_{n} w_{n}^{2}\right\}\right]
$$

To complete the specification of this hierarchical prior for $\boldsymbol{\alpha}$, the Gamma distribution is suitable [14]:

$$
p\left(\boldsymbol{\alpha} \mid c_{0}, d_{0}\right)=\prod_{n=1}^{N} \operatorname{Gamma}\left(\alpha_{n} \mid c_{0}, d_{0}\right)=\frac{d_{0}^{N c_{0}}}{\left(\Gamma\left(c_{0}\right)\right)^{N}} \cdot \prod_{n=1}^{N} \alpha_{n}^{c_{0}-1} \cdot \exp \left(-d_{0} \sum_{n=1}^{N} \alpha_{n}\right)
$$

To make this prior non-informative (i.e. almost flat), we set its parameters as: $c_{0}=1$ and $d_{0}=10^{-10}$, which makes it a very broad Exponential prior.

\subsection{BCS algorithm with MAP estimation of hyper-parameters $\alpha$ and $\beta$}

The following theory and BCS algorithm is based on [18] except for the modified prior PDF in (8b) that now is conditional on $\beta$, as well as $\boldsymbol{\alpha}$.

\subsubsection{Bayesian updating for the basis coefficients of the signal model}

The likelihood function in (6) for the CS measurements $\mathbf{y}$ and the prior on $\mathbf{w}$ in (8b) define a stochastic model class [29] $\mathcal{M}(\boldsymbol{\alpha}, \beta)$ for the signal model, which has the posterior distribution $p(\mathbf{w} \mid \mathbf{y}, \boldsymbol{\alpha}, \beta)$ given by Bayes' theorem: 


$$
p(\mathbf{w} \mid \mathbf{y}, \boldsymbol{\alpha}, \beta)=p(\mathbf{y} \mid \mathbf{w}, \beta) p(\mathbf{w} \mid \boldsymbol{\alpha}, \beta) / p(\mathbf{y} \mid \boldsymbol{\alpha}, \beta)=\mathcal{N}(\mathbf{w} \mid \boldsymbol{\mu}, \boldsymbol{\Sigma})
$$

with posterior mean and covariance matrix:

$$
\begin{aligned}
& \boldsymbol{\mu}=\mathbf{C}^{-1} \mathbf{\Theta}^{T} \mathbf{y}, \\
& \boldsymbol{\Sigma}=\beta^{-1} \mathbf{C}^{-1},
\end{aligned}
$$

In (10), the normalizing constant for the posterior PDF is the evidence (or marginal likelihood) for $\mathcal{M}(\boldsymbol{\alpha}, \beta)$ :

$$
p(\mathbf{y} \mid \boldsymbol{\alpha}, \beta)=\int p(\mathbf{y} \mid \mathbf{w}, \beta) p(\mathbf{w} \mid \boldsymbol{\alpha}, \beta) \mathbf{d} \mathbf{w}=\mathcal{N}\left(\mathbf{y} \mid 0, \beta^{-1} \mathbf{B}\right)
$$

where

$$
\mathbf{B}=\mathbf{I}_{K}+\boldsymbol{\Theta} \mathbf{A}^{-1} \boldsymbol{\Theta}^{T}
$$

The posterior probability distribution for the reconstructed signal $\mathbf{x}=\mathbf{\Psi} \mathbf{w}$ is also Gaussian, $p(\mathbf{x} \mid \mathbf{y}, \boldsymbol{\alpha}, \beta)=$ $\mathcal{N}\left(\mathbf{x} \mid \boldsymbol{\mu}_{\mathbf{x}}, \boldsymbol{\Sigma}_{\mathbf{x}}\right)$ with mean and covariance matrix: $\boldsymbol{\mu}_{\mathbf{x}}=\boldsymbol{\Psi} \boldsymbol{\mu}=\boldsymbol{\Psi} \mathbf{C}^{-1} \boldsymbol{\Theta}^{T} \mathbf{y}$ and $\boldsymbol{\Sigma}_{\mathbf{x}}=\boldsymbol{\Psi} \boldsymbol{\Sigma} \boldsymbol{\Psi}^{T}=\beta^{-1} \boldsymbol{\Psi C}^{-1} \boldsymbol{\Psi}^{T}$.

Inspired by [18], we suppose that the likelihood $p(\mathbf{y} \mid \boldsymbol{\alpha}, \beta)$ in (13) has a single pronounced global maximum with respect to the hyper-parameters $\boldsymbol{\alpha}$ and $\beta$, and so, therefore, does the posterior $\operatorname{PDF} \mathrm{p}(\boldsymbol{\alpha}, \beta \mid \mathbf{y})$ at $[\widehat{\boldsymbol{\alpha}}, \hat{\beta}]$ (the MAP value of $\boldsymbol{\alpha}$ and $\beta$ ). Then the unconditional posterior PDF $p(\mathbf{w} \mid \mathbf{y})$ can be estimated accurately using the most probable model class $\mathcal{M}(\widehat{\boldsymbol{\alpha}}, \hat{\beta})$ because of Laplace's asymptotic approximation [29,30,]:

$$
\begin{aligned}
p(\mathbf{w} \mid \mathbf{y}) & =\int p(\mathbf{w} \mid \mathbf{y}, \boldsymbol{\alpha}, \beta) p(\boldsymbol{\alpha}, \beta \mid \mathbf{y}) d \boldsymbol{\alpha} d \beta \\
& \approx p(\mathbf{w} \mid \mathbf{y}, \widehat{\boldsymbol{\alpha}}, \hat{\beta})
\end{aligned}
$$

where

$$
(\widehat{\boldsymbol{\alpha}}, \hat{\beta})=\arg \max _{[\boldsymbol{\alpha}, \beta]} p(\boldsymbol{\alpha}, \beta \mid \mathbf{y}) .
$$

\subsubsection{MAP estimation of hyper-parameters}

We first present an algorithm that is based on the MAP values of all of the uncertain hyper-parameters: $\boldsymbol{\alpha}, \beta, a_{0}$ and $b_{0}$, which are modeled as mutually independent a priori. By taking the Exponential prior on $a_{0}$ and $b_{0}$ and the Gamma prior on $\beta$ and $\boldsymbol{\alpha}$, we need to maximize the posterior $\operatorname{PDF} p\left(\boldsymbol{\alpha}, \beta, a_{0}, b_{0} \mid \mathbf{y}\right) \propto p(\mathbf{y} \mid \boldsymbol{\alpha}, \beta) p\left(\beta \mid a_{0}, b_{0}\right) p\left(\boldsymbol{\alpha} \mid c_{0}, d_{0}\right) p\left(a_{0}\right) p\left(b_{0}\right) \approx p(\mathbf{y} \mid \boldsymbol{\alpha}, \beta) p\left(\beta \mid a_{0}, b_{0}\right)$ since the priors on $\boldsymbol{\alpha}, a_{0}$ and $b_{0}$ are almost flat, where the evidence $p(\mathbf{y} \mid \boldsymbol{\alpha}, \beta)$ is given in (13).

This optimization can be done analytically to get the MAP value $\hat{\beta}$ :

$$
\hat{\beta}=\frac{K-2}{\mathbf{y}^{T} \mathbf{B}^{-1} \mathbf{y}}
$$

The MAP values $\hat{a}_{0}$ and $\hat{b}_{0}=\hat{a}_{0} \hat{\beta}^{-1}$ are not needed.

Since $p(\boldsymbol{\alpha})$ is taken as almost constant over the important region of the $\boldsymbol{\alpha}$ space, the optimization over $\boldsymbol{\alpha}$ 
to find its MAP value is essentially the same as maximizing the log of the evidence in $(13): \mathcal{L}(\boldsymbol{\alpha}, \beta)=$ $\log p(\mathbf{y} \mid \boldsymbol{\alpha}, \beta)=\log \mathcal{N}\left(\mathbf{y} \mid 0, \beta^{-1} \mathbf{B}\right)$. For this purpose we use an algorithm from [15] that is called the Bottom-up SBL algorithm in [18]. It starts with no terms in the basis expansion and then adds relevant ones to the signal model as the iterations proceed; this is done by updating a single hyper-parameter $\alpha_{n}$ at each iteration to monotonically increase the evidence. The algorithm is derived by isolating the contribution of each single hyper-parameter $\alpha_{n}$ in the $\log$ evidence function $\mathcal{L}(\boldsymbol{\alpha}, \beta)$ in a convenient form: $\mathcal{L}(\boldsymbol{\alpha}, \beta)=\mathcal{L}\left(\boldsymbol{\alpha}_{-n}, \beta\right)+l\left(\alpha_{n}, \beta\right)$, where $\mathcal{L}\left(\boldsymbol{\alpha}_{-n}, \beta\right)$ is the $\log$ evidence with the component $\alpha_{n}$ removed. Setting the derivative of $l\left(\alpha_{n}, \beta\right)$ with respect to $\alpha_{n}$ equal to zero leads to:

$$
\hat{\alpha}_{n}= \begin{cases}\infty, & \text { if } \hat{\gamma}_{n} \leq 0 \\ \hat{\gamma}_{n,} & \text { if } \hat{\gamma}_{n}>0\end{cases}
$$

where

$$
\hat{\gamma}_{n}=\frac{s_{n}^{2}}{\beta q_{n}^{2}-s_{n}}
$$

and the 'sparseness factor' $s_{n}$ and 'quality factor' $q_{n}$ are defined by:

$$
\begin{gathered}
s_{n}=\boldsymbol{\Theta}_{n}^{T} \mathbf{B}_{-n}^{-1} \boldsymbol{\Theta}_{n} \\
q_{n}=\mathbf{\Theta}_{n}^{T} \mathbf{B}_{-n}^{-1} \mathbf{y}
\end{gathered}
$$

where $\mathbf{B}_{-n}$ is $\mathbf{B}$ with the contribution of basis vector $\boldsymbol{\Theta}_{n}$ removed [15]. Note that the calculation of $s_{n}$ and $q_{n}$ only requires the numerical inversion of an $N^{\prime} \times N^{\prime}$ matrix, where $N^{\prime}$ is the number of non-zero terms in the current signal model and it is much smaller than the number of compressed measurements $K$ in this strategy. In addition, $s_{n}$ and $q_{n}$ are independent of $\alpha_{n}$, so we can find the stationary point of $l\left(\alpha_{n}, \beta\right)$ without iterative re-estimation. To study the nature of these stationary points, we also consider the second derivatives as shown in Appendix A. Finally, only the components that have finite $\alpha_{n}$ are retained in the signal model since each $w_{n}$ with $\alpha_{n}=\infty$ has prior mean and variance both zero, giving $w_{n}=0$, and so its term drops out of the signal model $\mathbf{x}=\Psi \mathbf{w}$.

\subsubsection{Algorithm BCS-MPE}

Notice that the optimal $\hat{\beta}$ in (15) depends on $\boldsymbol{\alpha}$ through $\mathbf{B}$ and the optimal $\widehat{\boldsymbol{\alpha}}$ from (16) depends on $\beta$ through $\hat{\gamma}_{n}$. Therefore, an iterative scheme is required for the full optimization of the evidence with respect to $[\alpha, \beta]$. We have found that some care is needed because of the important influence of $\beta$ on the evidence function but that successive relaxation works well [18]: first $\alpha$ is optimized with $\beta$ fixed and then $\beta$ is optimized with $\boldsymbol{\alpha}$ fixed at its intermediate optimal value, with this procedure being repeated until convergence is achieved. To initialize the algorithm, we select the single $\alpha_{n}$ of the $n^{\text {th }}$ term that 
maximizes $\left\|\boldsymbol{\Theta}_{n}^{T} \mathbf{y}\right\|^{2} /\left\|\boldsymbol{\Theta}_{n}\right\|^{2}$ over $n$ as $\alpha_{n}=1$. All other $\alpha_{n}{ }^{\prime} s$ are set to infinity, implying that the corresponding terms are excluded in the initial signal model. We update the prediction error precision $\beta$ based on this signal model, then fix this updated $\beta$ and optimize the intermediate evidence function to obtain a set of improved optimal $\alpha_{n}{ }^{\prime} s$.

The idea of successive relaxation produces a Bayesian CS reconstruction method that iterates between inner and outer loop optimizations. The outer loop updates the prediction-error precision $\beta$ and is terminated when the changes in the reconstructed signal models are sufficiently small, e.g. $\left\|(\widehat{\mathbf{x}})^{[j+1]}-(\widehat{\mathbf{x}})^{[j]}\right\|_{2}^{2} /\left\|(\widehat{\mathbf{x}})^{[j]}\right\|_{2}^{2}<\epsilon$, a specified threshold (we take $\epsilon=0.1$ in the examples later to avoid excessive numbers of iterations). The inner loop performs the optimization procedure over the hyper-parameter $\boldsymbol{\alpha}$ and is terminated when the change in all $\log \alpha_{n}{ }^{\prime} \mathrm{s}$ is less than $10^{-6}$. We call this procedure Algorithm BCS-MPE where 'MPE' denotes MAP estimation of the prediction-error parameter $\beta$.

In the inner loop, adding, deleting or re-estimating a basis vector $\boldsymbol{\Theta}_{n}$ in each iteration is based on whatever gives the maximum $\log$ evidence increase $\Delta \mathcal{L}_{n}$ [15]. To make the comparison, we need to calculate the increase of $\log$ evidence $\Delta \mathcal{L}_{n}$ for each term in the inner loop. Based on the isolated contribution of the $n^{\text {th }}$ term in the evidence function, it is estimated as $\Delta \mathcal{L}_{n}=l\left(\hat{\alpha}_{n}, \hat{\beta}\right)-l\left(\alpha_{n}, \hat{\beta}\right)$, where $\alpha_{n}$ and $\hat{\alpha}_{n}$ are the hyper-parameter value before optimization and after optimization, respectively, and $\hat{\beta}$ is the prediction error from the previous outer loop (see Steps 4-14 below). More efficient updating formulae with reduced computation that avoid any matrix inversions are given in Appendix A. The updated posterior mean $\widehat{\boldsymbol{\mu}}$ and covariance matrix $\widehat{\boldsymbol{\Sigma}}$ contain only those $N^{\prime}<N$ basis terms that are currently included in the signal model, and the computation thus involves only a small fraction of the full set of basis coefficients.

\section{Algorithm BCS-MPE}

1. Inputs: $\mathbf{\Theta}, \mathbf{y}$; Outputs: posterior mean and covariance of $\mathbf{w}$ and $\mathbf{x}$

2. Initialize all $\alpha_{n}^{\prime} s$ to a very large value except set $\alpha_{n}=1$ for the term that maximizes $\left\|\Theta_{n}^{T} \mathbf{y}\right\|^{2} /\left\|\Theta_{n}\right\|^{2}$ over $n=1, \ldots, N$

3. Compute initial $\boldsymbol{\mu}$ and $\boldsymbol{\Sigma}$ using (11) (both are scalars because $N^{\prime}=1$ initially), and initialize $s_{m}$ and $q_{m}$ for all terms $(m=1, \ldots, N)$, using the formulae in Appendix A.

4. While convergence criterion on the mean reconstructed signal model $\hat{\mathbf{x}}=\boldsymbol{\Psi} \boldsymbol{\mu}$ is not met 
(Outer loop)

5. Update $\beta$ based on the current $\alpha_{n}{ }^{\prime} s$ using (15)

6. While convergence criterion on the $\log \alpha_{n}{ }^{\prime} s$ is not met (Inner loop)

7. Select the basis vector $\boldsymbol{\Theta}_{n}$ with the largest log evidence increase $\Delta \mathcal{L}_{n}$

8. If $\beta q_{n}^{2}-s_{n}>0$ and $\alpha_{n}=\infty$, add $\boldsymbol{\Theta}_{n}$ and update $\alpha_{n}$ using (16)

9. If $\beta q_{n}^{2}-s_{n}>0$ and $\alpha_{n}<\infty$, re-estimate $\alpha_{n}$ using (16)

10. If $\beta q_{n}^{2}-s_{n} \leq 0$ and $\alpha_{n}<\infty$, delete $\boldsymbol{\Theta}_{n}$ and set $\alpha_{n}=\infty$

\section{End if}

12. Update $\boldsymbol{\mu}$ and $\boldsymbol{\Sigma}$, and $s_{m}$ and $q_{m}$ for all terms $(m=1, \ldots, N)$, using the formulae in Appendix A.

13. End while (the intermediate optimal $\alpha_{n}{ }^{\prime} s$ are then established for the current $\beta$ )

14. End while (the optimal hyper-parameters $\alpha$ and $\beta$ are then established)

For learning the hyper-parameters $\boldsymbol{\alpha}$ in Algorithm BCS-MPE, we note that in the maximization of the evidence function $p(\mathbf{y} \mid \boldsymbol{\alpha}, \beta)$ in (13), the objective function is not convex with respect to components $\alpha_{n}$ and there are local maxima that can trap an optimization, producing non-robust signal reconstructions. It is shown in Appendix $\mathrm{C}$ that the signal reconstruction of Algorithm BCS-MPE is robust for highly sparse signals if the successive relaxation is initialized with a very small prediction error precision $\beta$. The approximation in (14) is effective for CS reconstruction of highly sparse signals because the algorithmic optimization is likely to give the MAP values $(\widehat{\boldsymbol{\alpha}}, \hat{\beta})$.

It is also shown in Appendix $\mathrm{C}$ that there is a concern about the robustness for the BCS-MPE algorithm when applying it to approximately sparse signals. Although this trade-off between sparseness and data-fitting is always present, in the case of approximately sparse signals, the sensitivity of the evidence function to the uncertain prediction error precision $\beta$ may cause multiple peaks that are comparable to the global peak of the evidence function $p(\mathbf{y} \mid \boldsymbol{\alpha}, \beta)$. Using the BCS-MPE algorithm based on the MAP estimates of $(\boldsymbol{\alpha}, \beta)$ that maximize the evidence function $p(\mathbf{y} \mid \boldsymbol{\alpha}, \beta)$ may not lead to robust reconstructions because the optimization may become trapped in a local maximum, missing the other values of $(\boldsymbol{\alpha}, \beta)$ that produce larger contributions to the integral in Eq. (14) (that is, their evidence values are closer to the maximum value occurring at $(\widehat{\boldsymbol{\alpha}}, \hat{\beta})$ ).

The robust way to tackle this problem is to account for the full posterior uncertainty of the 
hyper-parameters $(\boldsymbol{\alpha}, \beta)$ by integrating them out as 'nuisance' parameters, that is, by marginalizing them. Although it turns out that marginalizing $\boldsymbol{\alpha} \in \mathbb{R}^{N}$ is analytically intractable, the prior $p\left(\mathbf{w}, \beta \mid \boldsymbol{\alpha}, a_{0}, b_{0}\right)=p(\mathbf{w} \mid \boldsymbol{\alpha}, \beta) p\left(\beta \mid a_{0}, b_{0}\right)$ given by Eqs. (8b) and (7) is conjugate to the likelihood $p(\mathbf{y} \mid \mathbf{w}, \beta)$ given by Eq. (6) and so both $\mathbf{w}$ and $\beta$ can be integrated out analytically, which can improve robustness during the optimization of $\boldsymbol{\alpha}$.

In the next section, a sparse Bayesian learning algorithm is proposed that integrates out the uncertain prediction-error precision parameter $\beta$ to make the $\mathrm{BCS}$ decompression algorithm more robust. We believe this algorithm is a new contribution. Ji et al. [31] integrated out the prior uncertainty on $\beta$ but not the posterior uncertainty to get the marginal posterior for $\mathbf{w}$.

\subsection{BCS algorithm with marginalization of prediction error $\beta$ and MAP estimation of $\alpha$}

\subsubsection{Bayesian updating for the basis coefficients of the signal model}

The stochastic model class $\mathcal{M}\left(\boldsymbol{\alpha}, a_{0}, b_{0}\right)$ for the signal model investigated in this section is defined by the likelihood function in (6) and the prior on $(\mathbf{w}, \beta)$ given by the product of (7) and (8b). We first get the posterior PDF for $\beta$, which is readily obtained by Bayes' theorem using the likelihood in (13) and prior in (7):

$$
\begin{aligned}
& p\left(\beta \mid \mathbf{y}, \boldsymbol{\alpha}, a_{0}, b_{0}\right) \propto p(\mathbf{y} \mid \boldsymbol{\alpha}, \beta) p\left(\beta \mid a_{0}, b_{0}\right) \\
= & \frac{\beta^{a_{0}-1+\frac{K}{2}}}{(2 \pi)^{\frac{K}{2}}} \frac{b_{0} a_{0}}{\Gamma\left(a_{0}\right)}|\mathbf{B}|^{-\frac{1}{2}} \exp \left\{-\frac{\beta}{2} \mathbf{y}^{T} \mathbf{B}^{-1} \mathbf{y}-b_{0} \beta\right\} \propto \operatorname{Gamma}\left(\beta \mid a_{0}^{\prime}, b_{0}^{\prime}\right) .
\end{aligned}
$$

where from Eq. (20), it is obvious that the posterior PDF of $\beta$ is a Gamma distribution $\operatorname{Gamma}\left(\beta \mid a_{0}^{\prime}, b_{0}^{\prime}\right)$ with the shape and rate parameters:

$$
\begin{gathered}
a_{0}^{\prime}=a_{0}+K / 2 \\
b_{0}^{\prime}=b_{0}+\mathbf{y}^{\mathrm{T}} \mathbf{B}^{-1} \mathbf{y} / 2 .
\end{gathered}
$$

According to the Total Probability Theorem, we then get for the posterior $p\left(\mathbf{w} \mid \mathbf{y}, \boldsymbol{\alpha}, a_{0}, b_{0}\right)$ from (10) and (20):

$$
\begin{aligned}
& p\left(\mathbf{w} \mid \mathbf{y}, \boldsymbol{\alpha}, a_{0}, b_{0}\right)=\int p(\mathbf{w} \mid \mathbf{y}, \boldsymbol{\alpha}, \beta) p\left(\beta \mid \mathbf{y}, \boldsymbol{\alpha}, a_{0}, b_{0}\right) d \beta \\
& =\frac{\Gamma\left(a_{0}^{\prime}+K / 2\right)\left(a_{0}^{\prime} / b_{0}^{\prime}\right)^{1 / 2}}{\Gamma\left(a_{0}^{\prime}\right)\left(2 \pi a_{0}^{\prime}\right)^{K / 2}|\mathbf{C}|^{-1 / 2}}\left\{1+\frac{1}{2 a_{0}^{\prime}}\left[\frac{a_{0}^{\prime}}{b_{0}^{\prime}}\left(\mathbf{w}-\mathbf{C}^{-1} \mathbf{\Theta}^{T} \mathbf{y}\right)^{T} \mathbf{C}\left(\mathbf{w}-\mathbf{C}^{-1} \mathbf{\Theta}^{T} \mathbf{y}\right)\right]\right\}^{-K / 2} \\
& =\operatorname{St}\left(\mathbf{w} \mid \boldsymbol{\mu}, \frac{a_{0}^{\prime}}{b_{0}^{\prime}} \mathbf{C}, 2 a_{0}^{\prime}\right)
\end{aligned}
$$

where $\boldsymbol{\mu}$ and $\mathbf{C}$ are given by (11a) and (12). This multivariate form of Student's t-distribution has mean and covariance matrix: 


$$
\begin{gathered}
\mathbf{E}\left(\mathbf{w} \mid \mathbf{y}, \boldsymbol{\alpha}, a_{0}, b_{0}\right)=\boldsymbol{\mu}=\mathbf{C}^{-1} \boldsymbol{\Theta}^{T} \mathbf{y} \\
\mathbf{C o v}\left(\mathbf{w} \mid \mathbf{y}, \boldsymbol{\alpha}, a_{0}, b_{0}\right)=\frac{b_{0}^{\prime}}{a_{0}^{\prime}-1} \mathbf{C}^{-1}
\end{gathered}
$$

It follows from $\mathbf{x}=\boldsymbol{\Psi} \mathbf{w}$ that for given hyper-parameters $\boldsymbol{\alpha}, a_{0}$ and $b_{0}$, the posterior probability distribution for the reconstructed signal $\mathbf{x}$ is a Student's t-distribution with mean and covariance matrix given by:

$$
\begin{gathered}
\mathbf{E}\left(\mathbf{x} \mid \mathbf{y}, \boldsymbol{\alpha}, a_{0}, b_{0}\right)=\boldsymbol{\Psi} \boldsymbol{\mu}=\boldsymbol{\Psi} \mathbf{C}^{-1} \Theta^{T} \mathbf{y} \\
\operatorname{Cov}\left(\mathbf{x} \mid \mathbf{y}, \boldsymbol{\alpha}, a_{0}, b_{0}\right)=\frac{b_{0}^{\prime}}{a_{0}^{\prime}-1} \boldsymbol{\Psi C}^{-1} \Psi^{T} .
\end{gathered}
$$

If the likelihood $p\left(\mathbf{y} \mid \boldsymbol{\alpha}, a_{0}, b_{0}\right)$, and so the posterior PDF $\mathrm{p}\left(\boldsymbol{\alpha}, a_{0}, b_{0} \mid \mathbf{y}\right)$, has a single pronounced global maximum with respect to $\boldsymbol{\alpha}, a_{0}$ and $b_{0}$, then the posterior PDF $p(\mathbf{w} \mid \mathbf{y})$ of interest can be estimated as [29,30]:

$$
\begin{aligned}
p(\mathbf{w} \mid \mathbf{y}) & =\int p\left(\mathbf{w} \mid \mathbf{y}, \boldsymbol{\alpha}, a_{0}, b_{0}\right) p\left(\boldsymbol{\alpha}, a_{0}, b_{0} \mid \mathbf{y}\right) d \boldsymbol{\alpha} d a_{0} d b_{0} \\
& \approx p\left(\mathbf{w} \mid \mathbf{y}, \widehat{\boldsymbol{\alpha}}, \hat{a}_{0}, \hat{b}_{0}\right)
\end{aligned}
$$

where $\left(\widehat{\boldsymbol{\alpha}}, \hat{a}_{0}, \hat{b}_{0}\right)=\arg \max _{\left[\boldsymbol{\alpha}, a_{0}, b_{0}\right]} p\left(\boldsymbol{\alpha}, a_{0}, b_{0} \mid \mathbf{y}\right)=\arg \max _{\left[\boldsymbol{\alpha}, a_{0}, b_{0}\right]}\left\{p\left(\mathbf{y} \mid \boldsymbol{\alpha}, a_{0}, b_{0}\right)\right\}$ (because of the almost uniform prior for $\boldsymbol{\alpha}, a_{0}$ and $\left.b_{0}\right)$.

\subsubsection{MAP estimation of the hyper-parameters}

To find the MAP values of the hyper-parameters $\left[\boldsymbol{\alpha}, a_{0}, b_{0}\right]$, we need the corresponding evidence function, which is given by:

$$
\begin{aligned}
& p\left(\mathbf{y} \mid \boldsymbol{\alpha}, a_{0}, b_{0}\right)=\int p(\mathbf{y} \mid \boldsymbol{\alpha}, \beta) p\left(\beta \mid a_{0}, b_{0}\right) d \beta \\
& =\frac{\Gamma\left(a_{0}+K / 2\right)\left(a_{0} / b_{0}\right)^{1 / 2}}{\Gamma\left(a_{0}\right)\left(2 \pi a_{0}\right)^{K / 2}|\mathbf{B}|^{1 / 2}}\left\{1+\frac{1}{2 a_{0}}\left(\frac{a_{0}}{b_{0}} \mathbf{y}^{T} \mathbf{B}^{-1} \mathbf{y}\right)\right\}^{-K / 2-a_{0}} \\
= & \operatorname{St}\left(\mathbf{y} \mid \mathbf{0}, \frac{a_{0}}{b_{0}} \mathbf{B}^{-1}, 2 a_{0}\right)
\end{aligned}
$$

Since $p(\boldsymbol{\alpha}), p\left(a_{0}\right)$ and $p\left(b_{0}\right)$ are taken as almost constant over the important regions of their parameter spaces, the optimization over $\boldsymbol{\alpha}, a_{0}$ and $b_{0}$ to find their MAP values is essentially the same as maximizing the $\log$ evidence function $\mathcal{L}\left(\boldsymbol{\alpha}, a_{0}, b_{0}\right)=\log p\left(\mathbf{y} \mid \boldsymbol{\alpha}, a_{0}, b_{0}\right)$. By solving the stationarity equations obtained from direct differentiation of $\mathcal{L}\left(\boldsymbol{\alpha}, a_{0}, b_{0}\right)$ with respect to $a_{0}$ and $b_{0}$, the optimal estimates $\tilde{a}_{0}$ and $\tilde{b}_{0}$ are given by:

$$
\begin{gathered}
\tilde{b}_{0}=\frac{\tilde{a}_{0}}{K} \mathbf{y}^{\mathrm{T}} \mathbf{B}^{-1} \mathbf{y} \\
\psi\left(\tilde{a}_{0}+K / 2\right)-\psi\left(\tilde{a}_{0}\right)-\log \left(1+\mathbf{y}^{\mathrm{T}} \mathbf{B}^{-1} \mathbf{y} / 2 \tilde{b}_{0}\right)=0
\end{gathered}
$$

The solution of these equations leads to $\tilde{a}_{0} \rightarrow \infty$. However, from (21a), we see that the effect of $K$ 
measurement points is to increase the value of the coefficient $a_{0}^{\prime}$ by $K / 2$. Thus, we can consider the parameter $a_{0}$ in the prior in terms of $2 a_{0}$ 'effective' prior measurements. Since our current knowledge of the true prediction error is little, the best choice is to make the shape parameter $a_{0}$ much smaller than $K / 2$, so that the prior distribution of $\beta$ has little effect on its posterior distribution. If we impose only the constraint of mean $\mathbf{E}\left(\beta \mid b_{0}\right)=1 / b_{0}$, the maximum entropy prior for $\beta$ is the exponential distribution, which is a special case of the Gamma distribution with shape parameter $a_{0}=1$. Since the maximum entropy prior PDF gives the largest uncertainty for $\beta$, subject to only the mean constraint, we choose it, and so set $\tilde{a}_{0}=1$ and then use (26) for the optimal value $\tilde{b}_{0}$. In the following, we denote $\mathcal{L}\left(\boldsymbol{\alpha}, \tilde{a}_{0}, \tilde{b}_{0}\right)$ by $\mathcal{L}(\boldsymbol{\alpha})$.

To analyze the dependence of $\mathcal{L}(\boldsymbol{\alpha})$ on a single hyper-parameter $\alpha_{n}$, we rewrite the log evidence $\mathcal{L}(\boldsymbol{\alpha})=\mathcal{L}\left(\boldsymbol{\alpha}_{-n}\right)+l\left(\alpha_{n}\right)$. where $\mathcal{L}\left(\boldsymbol{\alpha}_{-n}\right)$ is the log evidence with the component $\alpha_{n}$ removed. Setting the derivative of $l\left(\alpha_{n}\right)$ with respect to $\alpha_{n}$ equal to zero leads to the optimal values (see Appendix B for the stationary points):

$$
\tilde{\alpha}_{n}= \begin{cases}\infty, & \text { if } \tilde{\gamma}_{n} \leq 0 \\ \tilde{\gamma}_{n} & \text { if } \tilde{\gamma}_{n}>0\end{cases}
$$

where

$$
\tilde{\gamma}_{n}=\frac{s_{n}^{2}-s_{n} q_{n}^{2} / g_{n}}{\left(K+2 \tilde{a}_{0}\right) q_{n}^{2} / g_{n}-s_{n}}
$$

and $s_{n}$ and $q_{n}$ are given by (18) and (19), respectively,

$$
g_{n}=\mathbf{y}^{T} \mathbf{B}_{-n}^{-1} \mathbf{y}+2 \tilde{b}_{0}
$$

and

$\mathbf{y}^{\mathrm{T}} \mathbf{B}_{-n}^{-1} \mathbf{y}=\mathbf{y}^{\mathrm{T}}\left(\mathbf{I}_{K}+\boldsymbol{\Theta} \mathbf{A}_{-n}^{-1} \boldsymbol{\Theta}^{T}\right)^{-1} \mathbf{y}=\mathbf{y}^{\mathrm{T}}\left(\mathbf{I}_{K}-\boldsymbol{\Theta}\left(\mathbf{A}_{-n}+\boldsymbol{\Theta}^{T} \boldsymbol{\Theta}\right)^{-1} \boldsymbol{\Theta}^{T}\right) \mathbf{y}=\mathbf{y}^{\mathrm{T}}\left(\mathbf{y}-\boldsymbol{\Theta} \boldsymbol{\mu}_{-n}\right)$,

where $\mathbf{A}_{-n}$ and $\boldsymbol{\mu}_{-n}$ are $\mathbf{A}$ and $\boldsymbol{\mu}$ with $\alpha_{n}=\infty$ and $\mu_{n}=0$, respectively. Therefore, $\mathbf{y}^{\mathrm{T}} \mathbf{B}_{-n}^{-1} \mathbf{y}$ can be interpreted as a measure of the sensitivity of the prediction error to the exclusion of the $n^{\text {th }}$ term in the signal $\operatorname{model}\left(\alpha_{n}=\infty\right)$.

\subsubsection{Algorithm BCS-IPE}

Similar to the algorithm BCS-MPE, we employ successive relaxation to optimize the evidence in an iterative fashion to suppress the suboptimal solutions with too many nonzero terms: first, $b_{0}$ is updated using (26) with $\alpha_{n}=1$ for the term that maximizes $\left\|\boldsymbol{\Theta}_{n}^{T} \mathbf{y}\right\|^{2} /\left\|\boldsymbol{\Theta}_{n}\right\|^{2}$; then $\boldsymbol{\alpha}$ is optimized using (28)-(31) with $b_{0}$ fixed, followed by updating of $b_{0}$ using (26) with $\boldsymbol{\alpha}$ fixed at its current optimal value. This procedure is repeated 
until convergence is achieved. The procedure is summarized below in Algorithm BCS-IPE, where 'IPE' denotes integration over the prediction-error precision parameter $\beta$. The termination criteria for the inner and outer loops are the same as for BCS-MPE. The updating formulae in Appendix B are used to give a more efficient implementation that does not require any matrix inversions.

\section{Algorithm BCS-IPE}

1. Inputs: $\boldsymbol{\Theta}, \mathbf{y}$; Outputs: posterior mean and covariance of $\mathbf{w}$ and $\mathbf{x}$

2. Initialize all $\alpha_{n}{ }^{\prime} s$ to a very large value except set $\alpha_{n}=1$ for the term that maximizes $\left\|\Theta_{n}^{T} \mathbf{y}\right\|^{2} /\left\|\Theta_{n}\right\|^{2}$

3. Compute initial $\boldsymbol{\mu}$ and $\mathbf{C}$ using (11a) and (12) (both are scalars because $N^{\prime}=1$ initially), and initialize $s_{m}, q_{m}$ and $g_{m}$ for all terms $(m=1, \ldots, N)$, using the formulae in Appendix B.

4. While convergence criterion on the mean reconstructed signal model $\hat{\mathbf{x}}=\boldsymbol{\Psi} \boldsymbol{\mu}$ is not met (Outer loop)

5. Update parameter $b_{0}$ using (26) based on the current $\alpha_{n}{ }^{\prime} s$

6. While convergence criterion on the $\log \alpha_{n}{ }^{\prime} s$ is not met (Inner loop)

7. Select the basis vector $\boldsymbol{\Theta}_{n}$ with the largest log evidence increase $\Delta \mathcal{L}_{n}$

8. If $\tilde{\gamma}_{n}>0$ and $\alpha_{n}=\infty$, add $\boldsymbol{\Theta}_{n}$ and update $\alpha_{n}$ using (28)

9. If $\tilde{\gamma}_{n}>0$ and $\alpha_{n}<\infty$, re-estimate $\alpha_{n}$ using (28)

10. If $\tilde{\gamma}_{n} \leq 0$ and $\alpha_{n}<\infty$, delete $\boldsymbol{\Theta}_{n}$ and set $\alpha_{n}=\infty$

\section{End if}

12. Update $\boldsymbol{\mu}$ and $\mathbf{C}$, and $s_{m}, q_{m}$ and $g_{m}$ for all terms $(m=1, \ldots, N)$, using the formulae in Appendix B.

13. End while (the intermediate optimal $\alpha_{n}{ }^{\prime} s$ are then established for the current $b_{0}$ )

14. End while (the optimal hyper-parameters $\boldsymbol{\alpha}$ and $b_{0}$ are then established)

Remark 2.1: When $a_{0} \rightarrow \infty$, the prior PDF for $\beta$ in (7) tends to be a delta function $\delta\left(\beta-a_{0} / b_{0}\right)$, where the ratio of MAP estimates $\tilde{a}_{0} / \tilde{b}_{0}=\frac{K}{\mathbf{y}^{T^{-}} \mathbf{B}^{-1} \mathbf{y}}$ is close to $\hat{\beta}=\frac{K-2}{\mathbf{y}^{T} \mathbf{B}^{-1} \mathbf{y}}$ in (15) for larger $K$ values. Therefore, the stochastic model class $\mathcal{M}\left(\boldsymbol{\alpha}, a_{0}, b_{0}\right)$ investigated here becomes equivalent to the model class $\mathcal{M}(\boldsymbol{\alpha}, \beta)$ in 
Subsection 2.2, and the results from the BCS-IPE algorithm are essentially the same as those from the BCS-MPE algorithm. However, we argue in Appendix C that using the heavier-tailed multivariate Student-t distribution in (25) obtained by integrating over $\beta$, along with smaller value of $a_{0}$ (larger uncertainty in the prior PDF over $\beta$ ) reconstructs signals with greater robustness, especially when the original signal is approximately sparse. The numerical and experimental results given later support this conclusion because BCS-IPE outperforms BCS-MPE.

Although the full uncertainty in the prior PDF over $\beta$ is considered in the BCS-IPE algorithm, not just the MAP value used in BCS-MPE, the posterior uncertainty of the signal model parameter $\mathbf{w}$ is essentially the same when the hyper-parameter $\boldsymbol{\alpha}$ is given. We need to marginalize over the posterior PDF of $\beta$ to infer the marginal posterior of $\mathbf{w}$, as shown in (22), and the posterior covariance matrix in (23b) can be expressed as:

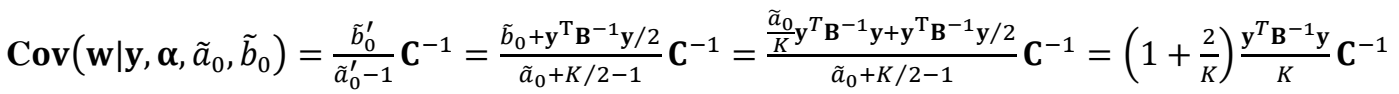

which is very close to $\operatorname{Cov}(\mathbf{w} \mid \mathbf{y}, \boldsymbol{\alpha}, \hat{\beta})=\hat{\beta}^{-1} \mathbf{C}^{-1}=\left(1-\frac{2}{K}\right)^{-1} \frac{\mathbf{y}^{T} \mathbf{B}^{-1} \mathbf{y}}{K} \mathbf{C}^{-1}$ in (11b) when $K$ is large.

Remark 2.2: Ji et al. [31] also marginalize the prediction-error parameter $\beta$ out. However, they integrate out the prior uncertainty on $\beta$ for inferring the marginal posterior PDF of the signal model parameter $\mathbf{w}$. We have shown that integration over the posterior uncertainty on $\beta$ is useful for getting more reliable posterior uncertainty quantification for $\mathbf{w}$. In their examples, they fix the shape parameter $a_{0}=(10 / \operatorname{std}(\mathbf{y}))^{2}$ and rate parameter $b_{0}=1$ in the Gamma prior PDF over $\beta$ while our algorithm has $a_{0}=1$ and optimizes over $b_{0}$ and $\boldsymbol{\alpha}$ by employing successive relaxation to maximize the evidence for the model class $\mathcal{M}\left(\boldsymbol{\alpha}, b_{0}\right)$, thereby updating all hyper-parameters effectively. The approach of $\mathrm{Ji}$ et al. [31] to marginalizing $\beta$ out does not lead to much improvement (e.g. see Fig. 4 in [31]).

\section{APPLICATIONS}

\subsection{Synthetic spike signals with various sparsity levels}

In this section we present numerical results of applying our proposed algorithms (BCS-MPE and BCS-IPE) to synthetic data and compare them to the results from two published Bayesian CS algorithms: BCS [12] and BCS-Laplace [13], and three state-of-the-art deterministic CS reconstruction algorithms: BP [1], GPSR [5], and AIHT [8]. For all these algorithms, MATLAB codes are downloaded from the corresponding websites [32-36] for BCS, BCS-Laplace, BP, GPSR and AIHT, respectively and the required parameters are set according to their 
default setups.

Consider test signals of length $N=512$, containing $T=20$ and $T=250$ nonzero spikes, which represent signals of high and low sparseness, respectively. The non-zero spikes are independent and identically distributed (i.i.d.) variables at randomly selected times with the amplitudes drawn from two different probability distributions: one uniform with equal probability of \pm 1 values and the other a zero-mean unit-variance Gaussian. For signal compression, a $K \times N$ zero-mean Gaussian random projection matrix with its columns scaled to give unit 2-norm is employed in each experiment and for the associated decompression, the reconstruction errors are calculated as $\|\widehat{\mathbf{w}}-\mathbf{w}\|_{2}^{2} /\|\mathbf{w}\|_{2}^{2}$, where $\widehat{\mathbf{w}}$ and $\mathbf{w}$ are the reconstructed (mean values for BCS algorithms) and original signal vectors, respectively.

Fig. 1(a-d)(i) shows four original signals of length $N=512$ and $T=20$ and 250 uniform and non-uniform (Gaussian) spikes. Fig. 1(a-d)(ii) and (iii) display the corresponding mean reconstruction results for BCS-MPE and BCS-IPE, respectively, for various lengths $K$ of the compressed data. It is seen that both methods can reconstruct the underlying highly sparse signal exactly $(T=20)$ (Fig. 1(a,b)) even for highly compressed data. However, BCS-MPE cannot recover the underlying approximately sparse signal $(T=250)$ well, especially for the signal with uniform spikes (Fig. 1(c)(ii)).

We then investigate the effect of different choices of compression ratios $(C R)$ on the signal reconstruction performance for different algorithms. In the experiments, we vary the number of measurements $K$ from 40 to 120 (compression ratios $C R=N / K$ from 4.27 to 12.8 ) and 280 to 460 (compression ratios $C R=N / K$ from 1.11 to 1.83) for signals with $T=20$ and $T=250$, respectively. Because of the randomness of the projection matrices in compressive sensing, we repeat the experiments 100 times with different projection matrices and report the overall performance. We employ two different thresholds of acceptable reconstruction error $(0.02$ and $0.20)$ and compute the corresponding rates of acceptable reconstruction.

In Figure 2, we present results for the noise level corresponding to added zero-mean Gaussian noise with standard deviation $0.001 \%$ of the RMS of the synthetic compressed measurements $\mathbf{y}$, since the measurement noise in (2) associated with the projection operation is expected to be negligible. The top row in each of Figures 2(a)-(d) compares our proposed BCS algorithms with two existing ones, while the bottom row compares our BCS-IPE with the three deterministic CS algorithms. As expected, increases in $C R$ beyond a critical value correspond to a decrease in the rates of acceptable performance for all methods and the higher sparseness level for a signal with $T=20$ non-zero components compared with that of $T=250$ corresponds to a significant 
increase in the critical value of $C R$. In addition, it is seen that higher diversity of the weight magnitudes (non-uniform spikes) tends to gives a larger rate of acceptable performance (e.g. compare Figure 2 (a) and (b)), which is consistent with the conclusion in [17] and [18].

For all of the results with different signal sparsity and spike diversity, it is seen that BCS-IPE algorithm has the largest critical value of compression ratio below which nearly perfect lossless reconstruction performance (RE<0.02) occurs. In fact, the BCS-IPE curves for the two different RE thresholds (0.02 and 0.2$)$ are essentially identical, so BCS-IPE is much more robust at giving very good reconstructions for both highly and approximately sparse signals. When the reconstruction error threshold is 0.2 and the signal is highly sparse $(T=20)$, BCS-MPE has slightly higher acceptance rates than BCS-IPE. This is expected since the marginalization over prediction error precision $\beta$ has no significant effect on the increase of signal reconstruction robustness as discussed in Appendix C. However, BCS-IPE clearly outperforms BCS-MPE for reconstructing approximately sparse signals $(T=250)$, which is shown by its much higher rates of acceptable performance, which is consistent with our remarks in Section 2.3 that are supported by the analyses in Appendix C.

In Table 1, average running times for producing the reconstructed signals in Figure 2 are shown. It is clear that the proposed BCS-IPE and BCS-MPE methods are slower than other methods because of the sucessive relaxation procedure employed to learn all uncertain parameters. However, this procedure has the advantage that there is no user intervention required to set parameters related to signal sparseness, noise levels, etc, which is necessary for the deterministic algorithms and the Bayesian algorithms BCS and BCS-Lap. In addition, the automatic learning of all parameters in BCS-IPE and BCS-MPE produce better signal reconstruction performance than the other investigated methods, as shown in Figure 2. Therefore, the choice of CS reconstruction methods in real applications is a trade-off between the level of accuracy that the user is willing to accept and the computation time.

Compared with the deterministic CS reconstruction algorithms (BP, GPSR and AIHT), the Bayesian CS methods allow quantification of the posterior uncertainty, or confidence, for the reconstructed signals. For this purpose, the "error bars" can be computed as the square root of the diagonal elements of the posterior covariance matrix given in (33). In Figure 3, the reconstruction errors and average error bars for 50 trials with different projection matrices but the same original signal are shown for the four BCS algorithms. The average error bars are calculated over all nonzero terms in each signal model. The number of measurements $K$ is selected 
as small as possible for robust reconstructions, and differs for each signal ( $K=90,60,470$ and 380 for signals in Figure 3(a), (b), (c) and (d), respectively). The added noise level is $0.001 \%$.

It is seen that for BCS-IPE (Figure 3 (a-d)(iv)), the average error bars of correctly reconstructed signals (with RE essentially zero) are much smaller than those of BCS-MPE (Figure 3(a-d)(iii)), which indicates higher confidence in the signal reconstruction and leads to a pleasing consequence that all incorrect (suboptimal) reconstructed signal models for BCS-IPE give much larger average error bars than those of correct (optimal) reconstruction cases. Therefore, the estimated error bars for BCS-IPE provide a useful tool for signal reconstruction diagnostics without knowing the original signal. For other methods, the correspondence of larger error bars with suboptimal reconstructions (larger RE values) is not discernible, especially for approximately sparse signals (Figure 3(c-d)(i-iii)), which may lead to a false judgement about the confidence of the signal reconstructions.

If there are no strict demands on computation time, the BCS-IPE method provides the best overall performance among all methods considering RE and uncertainty quantification of the results, especially for approximately sparse signals.

\subsection{Real structural health monitoring signals}

We now examine the performance of our proposed BCS methods for real accelerometer data from the structural health monitoring (SHM) systems on two civil structures. Case 1 is the Beijing National Aquatics Center (Figure 4), popularly called the Water Cube, which is a well-known steel space-frame structure built for the 2008 Olympics swimming facility. A sophisticated long-term structural health monitoring system [37] was installed on this structure in 2008. An ambient vibration response signal of length 512 seconds (Figure 4) and sample frequency $100 \mathrm{~Hz}$ from one of the accelerometers is studied here. Case 2 is the Tianjin Yonghe Bridge [38] (Figure 5), which is one of the earliest cable-stayed bridges constructed in the mainland of China. An acceleration time history of length 512 seconds and sample frequency $100 \mathrm{~Hz}$ was selected from one of the accelerometers (see Figure 5).

Using the Haar wavelet transform, the wavelet coefficients of the acceleration signal from the Water Cube and Tianjin Yonghe Bridge are computed and shown in Figure 6(a) and (b), respectively, which reveals that the strict sparseness levels of the wavelet coefficient vectors are low. For the Water Cube, none of the wavelet coefficients are exactly zero but the $45 \%$ largest magnitude components contain $80 \%$ of the energy of the signal (in terms of the sum of square values). For Tianjin Yonghe Bridge, only $0.5 \%$ of the components have 
magnitude exactly zero. However, the effective sparseness level is quite high: the $16 \%$ largest magnitude components contain $80 \%$ of the energy of the signal. The different sparseness levels are a consequence of the different dynamic characteristics of the two structures.

We divide the signals in Figures 4 and 5 into 100 segments of length $N=512$ and compress the signal in each segment by projection using the same sample of a zero-mean Gaussian projection matrix $\boldsymbol{\Phi} \in \mathbb{R}^{K \times N}$ to get the compressed data $\mathbf{y}$. For the matrix $\boldsymbol{\Theta}=\boldsymbol{\Phi} \boldsymbol{\Psi}$ in (3), $\boldsymbol{\Psi}$ is the discrete orthonormal Haar wavelet basis matrix constructed using the MATLAB routine at http://gtwavelet.bme.gatech.edu/. For a real CS accelerometer, we would obtain data already in a compressed form, where the projection arithmetic is integrated with the analog-to-digital converter in the sensor itself, so the actual signal, denoted $\overline{\mathbf{x}}$ and its wavelet coefficients $\overline{\mathbf{w}}=$ $\boldsymbol{\Psi}^{T} \overline{\mathbf{X}}$, would be unknown. We investigate the reconstruction problem for the compressed measurement vector $\mathbf{y}=\boldsymbol{\Phi} \overline{\mathbf{x}}$ for Cases 1 and 2, corresponding to approximately sparse signals. In this case, measurement noise $\mathbf{r}$ in (2) is zero because the real signal always contains it and we do not add any synthetic noise.

For data decompression, we run the Bayesian CS algorithms to produce a probabilistic description of the reconstructed coefficients $\mathbf{w}$ with mean $\mathbf{E}(\mathbf{w} \mid \mathbf{y})$ from compressed measurements $\mathbf{y}$. The corresponding uncertain reconstructed acceleration signals $\mathbf{x}$ are then obtained by wavelet transforms: $\mathbf{x}=\boldsymbol{\Psi}_{\mathbf{w}}$. In the results shown later, the strict reconstruction-error measures for the signals recovered from the compressed measurements $\mathbf{y}$ are defined by:

$\mathbf{R E}=\|\overline{\mathbf{w}}-\mathbf{E}(\mathbf{w} \mid \mathbf{y})\|_{2}^{2} /\|\overline{\mathbf{w}}\|_{2}^{2}=\|\boldsymbol{\Psi} \overline{\mathbf{w}}-\mathbf{\Psi} \cdot \mathbf{E}(\mathbf{w} \mid \mathbf{y})\|_{2}^{2} /\|\boldsymbol{\Psi} \overline{\mathbf{w}}\|_{2}^{2}=\|\overline{\mathbf{x}}-\mathbf{E}(\mathbf{x} \mid \mathbf{y})\|_{2}^{2} /\|\overline{\mathbf{x}}\|_{2}^{2}$

where we have used the orthonormality of the wavelet basis, so $\boldsymbol{\Psi}^{T} \boldsymbol{\Psi}=\mathbf{I}_{N}$.

We expect the wavelet coefficients with larger amplitudes to constitute the desirable part of the original signal and be sparsely distributed. The CS reconstruction of the effective wavelet vector $\overline{\mathbf{w}}_{s}$ may therefore be of more interest; it contains only the $T_{s}$ wavelet coefficients with magnitudes significantly larger than the "noise" background. We introduce the index vector id to indicate the locations of the $T_{S}$ non-zero components in $\overline{\mathbf{w}}_{S}$. We also calculate the effective reconstruction-error measures for reconstructed $\mathbf{w}_{s}$ and corresponding time domain signal $\mathbf{x}_{s}=\boldsymbol{\Psi}_{\mathbf{w}_{s}}$ as:

$\mathbf{R E}_{s}=\left\|\overline{\mathbf{w}}_{s}-\mathbf{E}\left(\mathbf{w}_{s} \mid \mathbf{y}\right)\right\|_{2}^{2} /\left\|\overline{\mathbf{w}}_{s}\right\|_{2}^{2}=\left\|\overline{\mathbf{x}}_{s}-\mathbf{E}\left(\mathbf{x}_{s} \mid \mathbf{y}\right)\right\|_{2}^{2} /\left\|\overline{\mathbf{x}}_{s}\right\|_{2}^{2}$

where the nonzero components of $\mathbf{w}_{s}$ consist of the corresponding components in $\mathbf{w}$ for fixed index id (it is known in this test). 
In Figure 7, an example of reconstructed wavelet coefficients using Algorithm BCS-IPE on the first time segment of the Tianjin Yonghe Bridge signal (Case 2) is shown. The strict reconstruction error RE is 0.1995, which is too large to be acceptable for real applications. However, we observe that most of the wavelet coefficients in $\overline{\mathbf{w}}$ are minor. If we choose the first $1 / 16(T=32)$ and $1 / 4(T=128)$ wavelet coefficients in $\overline{\mathbf{w}}$ when listed in decreasing magnitude to constitute the non-zero coefficients in $\overline{\mathbf{w}}_{S}$, then the effective reconstruction-error measures $\mathbf{R E}_{\mathrm{s}}$ quantified by (41) become much smaller with values of $0.0605(T=32)$ and $0.1201(T=128)$, respectively, demonstrating that much more accurate reconstruction can be achieved for wavelets coefficients with larger amplitudes. In addition, the posterior error-bars for the BCS-IPE method are shown in Figure 7(c), which can be used to provide effective uncertainty quantification for the reconstructed wavelet coefficients.

Similar to Figure 2 in the example with synthetic signals, Figures 8-9 show the results for the proposed Bayesian algorithms (BCS-MPE and BCS-IPE) along with the corresponding results for BCS [12], BCS-Laplace [13], BP [1], GPSR [5], and AIHT [8]. We vary the number of compressed measurements $K$ from 250 to 470 (compression ratios $C R$ of $N / K$ from 1.09 to 2.05 , where $N=512$ ) for Case 1 (Figure 8 ) and from 170 to 470 (compression ratios $C R$ from 1.09 to 3.01) for Case 2 (Figures 9). Different thresholds (0.02 and 0.20) of acceptable reconstruction errors $\mathbf{R E}$ and $\mathbf{R E}_{s}$, as quantified in (40-41), are also employed to present the rates of acceptable performance for the 100 time segments, based on the results using different numbers of compressed measurements $K$. In these experiments, the first $1 / 16$ and $1 / 4$ largest magnitude wavelet coefficients in $\overline{\mathbf{w}}$ are selected to constitute the non-zero coefficients in $\overline{\mathbf{w}}_{S}$.

Comparing acceptance rates in different columns of Figures 8 and 9 for different levels of reconstruction error in Cases 1 and 2, it is seen that more reconstructions are acceptable if judged by only the largest effective wavelet coefficients, which is consistent with the conclusion found from Figure 7. Figure 9(a) shows that when the compression ratio $C R$ is smaller than about 1.5 for Case 2, all effective reconstruction error measures for the 1/16 largest magnitude wavelet coefficients are smaller than 0.2 for BP and all of the Bayesian CS methods. However, the corresponding plot for Case 1 in Figure 8(a) shows that only BCS-IPE and BP provides robust acceptable reconstructions but only for $C R$ up to 1.1 . For all wavelet coefficients, it is seen that no perfect reconstruction is achieved for all methods because the sparseness levels are too low in the studied SHM signals, and so the posterior uncertainty quantification cannot be used here for diagnosis of the signal reconstruction accuracy, unlike Figure 3 for the synthetic signals. 
The observations above demonstrate that the proposed BCS-IPE provides the best overall performance among all methods. Therefore, if CS sensors are implemented in a wireless structural health monitoring system for signal compression, then Algorithm BCS-IPE is a viable candidate for reconstructing the original signal. However, a practical problem that needs to be addressed is that data loss may occur during wireless transmission from a sensor to the CPU $[24,25]$.

The signal reconstruction performance is investigated when there are $K_{m}$ data points lost in $\mathbf{y}=\mathbf{\Phi} \overline{\mathbf{x}}$ and the received data vector $\mathbf{y}_{l}$ contains only $K_{l}=K-K_{m}$ data points. Signal recovery is essentially the same as data decompression in CS: the corresponding $K_{m}$ rows of the projection matrix $\boldsymbol{\Phi} \in \mathbb{R}^{K \times N}$ are discarded to get a new matrix $\boldsymbol{\Phi}_{l} \in \mathbb{R}^{K_{l} \times N}$ that is, the received compressed measurement vector $\mathbf{y}_{l} \in \mathbb{R}^{K_{l}}$ is effectively produced by linear projections of the original signal $\overline{\mathbf{x}}$ using matrix $\boldsymbol{\Phi}_{l}, \mathbf{y}_{l}=\boldsymbol{\Phi}_{l} \overline{\mathbf{x}}$ (we assume that it is known which components of $\mathbf{y}$ are lost in transmission but not what their values are).

In a wireless sensor network, the data packets, each of which contains a certain number of data points, are transmitted one by one, and all the data points in a lost packet will be missing. In our data-recovery experiments, we assume four sampling points are included in each data packet and therefore 128 data packets are required for an uncompressed measurement $\mathbf{y}(K=N=512)$. For the purpose of examining the signal reconstruction performance of BCS-IPE for different data loss rates, we vary the number of lost data packets from 1 to 26 (data loss rate of $K_{l} / K$ from $0.78 \%$ to $20.31 \%$ ). The lost data packets are selected randomly among the 128 candidates, and we execute the same experiment 100 times and report the overall reconstruction performance.

Figure 10 is like Figures 8-9 except that it shows five different thresholds, 0.01, 0.02, 0.05, 0.10 and 0.20, of acceptable reconstruction errors to denote the rates of acceptable performance for the 100 runs for each possible data loss rate. It is observed that almost all reconstructions are acceptable if the threshold of acceptable strict reconstruction error measures is set to be 0.1 , as long as the data loss rates are smaller than $7 \%$ and $9 \%$, for Cases 1 and 2, respectively (Figure $10(\mathrm{e}, \mathrm{f})$ ). For relatively larger magnitude wavelet coefficients, it is seen that all reconstructions have effective reconstruction errors smaller than 0.05 and 0.10 when investigating the $1 / 16$ and 1/4 largest magnitude wavelet coefficients, respectively, and the data loss rate is less than $8 \%$ and 20\%, for Cases 1 and 2, respectively (Figure 10 (a,b,c,d)). These reconstruction errors are thought to be tolerable for structural modal identification and damage assessment using the reconstructed signals. BCS-IPE is therefore a promising algorithm for automated recovery of any data lost during wireless transmission, which can be used to guard against data loss even if the signal is not sparse in any basis. 


\section{CONCLUDING REMARKS}

Compressive sensing techniques have the potential to increase the efficiency of wireless data transmission and for data loss recovery. Most of the existing CS techniques allow effective reconstruction of signals only when they are sufficiently sparse in terms of some basis. However, signals in reality are usually only approximately sparse, so Bayesian CS for such signals is explored in this study for the first time.

From a robustness analysis based on an information-theoretic interpretation, it is found that the reconstruction of approximately-sparse signals is very sensitive to the selection of the prediction error precision $\beta$, which can lead to poor signal reconstructions by existing BCS methods that either fix the value of $\beta$ or estimate its MAP value. To enhance the signal reconstruction robustness for approximately sparse signals, we present the BCS-IPE algorithm for robust treatment of the prediction error precision $\beta$ where we marginalize out this parameter to effectively account for its prior and posterior uncertainties. We also employ successive relaxation to maximize the evidence function, thereby updating all hyper-parameters effectively. The effective dimensionality (number of nonzero basis coefficients) of the signal model is determined automatically as part of the hierarchical Bayesian inference procedure, and all uncertain model parameters are estimated solely from the compressed data and without any need for parameter tuning.

The superior performance of the proposed BCS-IPE algorithm in term of signal reconstruction robustness and posterior uncertainty quantification is demonstrated using both synthetic and real signals by comparing its performance with several state-of-the-art Bayesian and deterministic CS reconstruction methods. For the investigated real SHM signals, although the allowable compression ratios for reliable signal reconstructions are not so high because of their low sparseness, these ratios are sufficient to allow acceptable signal recovery of around 7-9\% loss of data during wireless transmission. It is also demonstrated that the reconstruction accuracy and posterior uncertainty quantification for wavelet coefficients with large amplitudes are much better than those with small amplitudes using the proposed BCS-IPE algorithm, allowing acceptable signal recovery for $10-15 \%$ loss of data. Such reconstructed signals are expected to allow reliable modal identification and damage assessment of a structure.

\section{Acknowledgements}

This work was supported by the U.S. National Science Foundation under award number EAR-0941374 to the California Institute of Technology. This support is gratefully acknowledged by the first two authors. This 
research is also supported by grant from the National Natural Science Foundation of China (NSFC grant no. 51308161) and the International Postdoctoral Exchange Fellowship Program 2014 by the Office of China Postdoctoral Council, which partially supported the first author and this support is also gratefully acknowledged.

Appendix A: Properties of stationary points and efficient updating formulae for hyper-parameter $\alpha_{n}$ for Algorithm BCS-MPE

The gradient of logarithm function $\mathcal{L}(\boldsymbol{\alpha}, \beta)$ in Section 2.2.2 is computed as:

$$
\frac{\partial \mathcal{L}(\boldsymbol{\alpha}, \beta)}{\partial \alpha_{n}}=\frac{\partial l(\boldsymbol{\alpha}, \beta)}{\partial \alpha_{n}}=\frac{\alpha_{n}^{-1} s_{n}^{2}-\left(\beta q_{n}^{2}-s_{n}\right)}{2\left(\alpha_{n}+s_{n}\right)^{2}}
$$

The stationary points occurs at both $\hat{\alpha}_{n}=\infty$ and $\hat{\alpha}_{n}=s_{n}^{2} /\left(\beta q_{n}^{2}-s_{n}\right)$ (subjected to $\beta q_{n}^{2}-s_{n}>0$ ).

To explore the property of the stationary points, we consider the second derivatives and substitute the point $\hat{\alpha}_{n}=s_{n}^{2} /\left(\beta q_{n}^{2}-s_{n}\right)$ to get:

$$
\left.\frac{\partial^{2} \mathcal{L}(\boldsymbol{\alpha}, \beta)}{\partial \alpha_{n}^{2}}\right|_{\widehat{\alpha}_{n}}=\frac{-s_{n}^{2}}{2 \widehat{\alpha}_{n}^{2}\left(\widehat{\alpha}_{n}+s_{n}\right)^{2}}
$$

It is seen that the second derivative $\frac{\partial^{2} \mathcal{L}}{\partial \alpha_{n}^{2}}$ is always negative at the point $\hat{\alpha}_{n}$, so $\hat{\alpha}_{n}$ gives the maximum of $\mathcal{L}(\boldsymbol{\alpha}, \beta)$ when $\beta$ is fixed and $\beta q_{n}^{2}-s_{n}>0$ so $\hat{\alpha}_{n}>0$. If $\beta q_{n}^{2}-s_{n} \leq 0$, then $\alpha_{n}=\infty$ gives the maximum.

The following efficient updating formulae for hyper-parameters $\alpha_{n}$ are based on [15] but for the prior PDF in (8b) instead of (8a).

In Step 12 of Algorithm BCS-MPE, it is required to update $\boldsymbol{\mu}$ and $\boldsymbol{\Sigma}$, and $s_{m}$ and $q_{m}$, for all terms ( $m=$ $1, \ldots, N)$. In the updating formulae, $s_{m}$ and $q_{m}$ are computed from:

$$
\begin{aligned}
& s_{m}=\mathcal{S}_{m} /\left(1-\alpha_{m}^{-1} \mathcal{S}_{m}\right) \\
& q_{m}=Q_{m} /\left(1-\alpha_{m}^{-1} \mathcal{S}_{m}\right)
\end{aligned}
$$

where the quantities $\mathcal{S}_{m}$ and $\mathcal{Q}_{m}$ are defined as:

$$
\begin{gathered}
\mathcal{S}_{m}=\boldsymbol{\Theta}_{m}^{T} \mathbf{B}^{-1} \boldsymbol{\Theta}_{m}=\boldsymbol{\Theta}_{m}^{T} \boldsymbol{\Theta}_{m}-\beta \boldsymbol{\Theta}_{m}^{T} \boldsymbol{\Theta} \boldsymbol{\Sigma} \boldsymbol{\Theta}^{T} \boldsymbol{\Theta}_{m} \\
\mathcal{Q}_{m}=\mathbf{\Theta}_{m}^{T} \mathbf{B}^{-1} \mathbf{y}=\boldsymbol{\Theta}_{n}^{T} \mathbf{y}-\beta \boldsymbol{\Theta}_{m}^{T} \boldsymbol{\Theta} \boldsymbol{\Sigma} \boldsymbol{\Theta}^{T} \mathbf{y}
\end{gathered}
$$

where $\boldsymbol{\Theta}_{m}$ is the $m^{\text {th }}$ column of $\boldsymbol{\Theta}$.

Efficient updating formulae for each potential implementation in Steps 7-12 of Algorithm BCS-MPE are given here and updated quantities are denoted by a hat (e.g. $\left.\hat{\alpha}_{n}\right)$. 
(1) If $\hat{\gamma}_{n}>0$ and $\alpha_{n}=\infty$, add $\boldsymbol{\Theta}_{n}$ in the model and update the corresponding $\alpha_{n}$, and:

$$
\begin{gathered}
\Delta \mathcal{L}=\frac{\beta Q_{n}^{2}-\delta_{n}}{2 \delta_{n}}+\frac{1}{2} \log \frac{s_{n}}{\beta Q_{n}^{2}} \\
\widehat{\boldsymbol{\Sigma}}=\left[\begin{array}{cc}
\boldsymbol{\Sigma}+\beta^{2} \Sigma_{n n} \boldsymbol{\Sigma} \boldsymbol{\Theta}^{T} \boldsymbol{\Theta}_{n} \boldsymbol{\Theta}_{n}^{T} \boldsymbol{\Theta} \boldsymbol{\Sigma} & -\beta^{2} \boldsymbol{\Sigma}_{n n} \boldsymbol{\Sigma} \boldsymbol{\Theta}^{T} \boldsymbol{\Theta}_{n} \\
-\beta^{2} \Sigma_{n n}\left(\boldsymbol{\Sigma} \boldsymbol{\Theta}^{T} \boldsymbol{\Theta}_{n}\right)^{T} & \boldsymbol{\Sigma}_{n n}
\end{array}\right] \\
\widehat{\boldsymbol{\mu}}=\left[\begin{array}{c}
\boldsymbol{\mu}-\beta \boldsymbol{\mu}_{n} \boldsymbol{\Sigma} \boldsymbol{\Theta}^{T} \boldsymbol{\Theta}_{n} \\
\boldsymbol{\mu}_{n}
\end{array}\right]
\end{gathered}
$$

where

$$
\boldsymbol{\Sigma}_{n n}=\beta^{-1}\left(\alpha_{n}+\mathcal{S}_{n}\right)^{-1}, \mu_{n}=\beta \Sigma_{n n} \mathcal{Q}_{n}
$$

For each term $m=1, \ldots, N$ :

$$
\begin{gathered}
\hat{\mathcal{S}}_{m}=\mathcal{S}_{m}-\beta \Sigma_{n n}\left(\boldsymbol{\Theta}_{m}^{T} \boldsymbol{e}_{n}\right)^{2} \\
\hat{\mathcal{Q}}_{m}=\mathcal{Q}_{m}-\mu_{n}\left(\boldsymbol{\Theta}_{m}^{T} \boldsymbol{e}_{n}\right)
\end{gathered}
$$

where we define

$$
\boldsymbol{e}_{n}=\boldsymbol{\Theta}_{n}-\beta \boldsymbol{\Theta} \boldsymbol{\Sigma} \boldsymbol{\Theta}^{T} \boldsymbol{\Theta}_{n}
$$

(2) If $\hat{\gamma}_{n}>0$ and $\alpha_{n}<\infty$, retain $\boldsymbol{\Theta}_{n}$ in the model and update the corresponding $\alpha_{n}$, and:

$$
\begin{gathered}
\Delta \mathcal{L}=\frac{\beta Q_{n}^{2}}{2\left(\delta_{n}+\left(\widehat{\alpha}_{n}^{-1}-\alpha_{n}^{-1}\right)^{-1}\right)}-\frac{1}{2} \log \left[1+\delta_{n}\left(\hat{\alpha}_{n}^{-1}-\alpha_{n}^{-1}\right)\right] ; \\
\widehat{\boldsymbol{\Sigma}}=\boldsymbol{\Sigma}-\vartheta_{j} \boldsymbol{\Sigma}_{j} \boldsymbol{\Sigma}_{j}^{T} \\
\widehat{\boldsymbol{\mu}}=\boldsymbol{\mu}-\vartheta_{j} \mu_{j} \boldsymbol{\Sigma}_{j}
\end{gathered}
$$

where we define $\vartheta_{j}=\left(\Sigma_{j j}+\beta^{-1}\left(\hat{\alpha}_{n}-\alpha_{n}\right)^{-1}\right)^{-1}, j$ denotes the index within the current basis (a smaller fraction of the full set of basis vectors) that corresponds to the single term $n$ to be updated, and $\boldsymbol{\Sigma}_{j}$ is the $j t h$ column of $\boldsymbol{\Sigma}$. For each term $m=1, \ldots, N$ :

$$
\begin{aligned}
& \hat{\mathcal{S}}_{m}=\mathcal{S}_{m}+\beta \vartheta_{j}\left(\boldsymbol{\Sigma}_{j}^{T} \boldsymbol{\Theta}^{T} \boldsymbol{\Theta}_{m}\right)^{2} \\
& \hat{\mathcal{Q}}_{m}=\mathcal{Q}_{m}+\vartheta_{j} \mu_{j}\left(\boldsymbol{\Sigma}_{j}^{T} \boldsymbol{\Theta}^{T} \boldsymbol{\Theta}_{m}\right)
\end{aligned}
$$

(3) If $\hat{\gamma}_{n} \leq 0$ and $\alpha_{n}<\infty$, delete $\boldsymbol{\Theta}_{n}$ from the model and update the corresponding $\alpha_{n}$, and:

$$
\begin{gathered}
\Delta \mathcal{L}=\frac{\beta Q_{n}^{2}}{2\left(\delta_{n}-\alpha_{n}\right)}-\frac{1}{2} \log \left(1-\delta_{n} \alpha_{n}^{-1}\right) . \\
\widehat{\boldsymbol{\Sigma}}=\boldsymbol{\Sigma}-\frac{\mathbf{1}}{\Sigma_{j j}} \boldsymbol{\Sigma}_{j} \boldsymbol{\Sigma}_{j}^{T}
\end{gathered}
$$




$$
\widehat{\boldsymbol{\mu}}=\boldsymbol{\mu}-\frac{\mu_{j}}{\Sigma_{j j}} \boldsymbol{\Sigma}_{j}
$$

Following updates (A18) and (A19), the appropriate row and column are removed from $\widehat{\boldsymbol{\Sigma}}$ and $\widehat{\boldsymbol{\mu}}$. For each term $m=1, \ldots, N$ :

$$
\begin{gathered}
\hat{\mathcal{S}}_{m}=\mathcal{S}_{m}+\frac{\beta}{\Sigma_{j j}}\left(\boldsymbol{\Sigma}_{j}^{T} \boldsymbol{\Theta}^{T} \boldsymbol{\Theta}_{m}\right)^{2} \\
\hat{\mathcal{Q}}_{m}=Q_{m}+\frac{\mu_{j}}{\Sigma_{j j}}\left(\boldsymbol{\Sigma}_{j}^{T} \boldsymbol{\Theta}^{T} \boldsymbol{\Theta}_{m}\right)
\end{gathered}
$$

Appendix B: Properties of stationary points and efficient updating formulae for hyper-parameter $\alpha_{n}$ for

\section{Algorithm BCS-IPE}

The derivative of the logarithm function $\mathcal{L}\left(\boldsymbol{\alpha}, a_{0}, b_{0}\right)$ of the evidence in (25) with respect to $\alpha_{n}$ is given by:

$$
\frac{\partial \mathcal{L}\left(\boldsymbol{\alpha}, a_{0}, b_{0}\right)}{\partial \alpha_{n}}=\frac{\partial l\left(\boldsymbol{\alpha}, a_{0}, b_{0}\right)}{\partial \alpha_{n}}=\frac{\alpha_{n}^{-1}\left(s_{n}^{2}-s_{n} q_{n}^{2} / g_{n}\right)-\left(K+2 a_{0}\right) q_{n}^{2} / g_{n}+s_{n}}{2\left(\alpha_{n}+s_{n}\right)\left(\alpha_{n}+s_{n}-q_{n}^{2} / g_{n}\right)}
$$

The stationary points are $\tilde{\alpha}_{n}=\infty$ and $\tilde{\alpha}_{n}=\tilde{\gamma}_{n}=\left(s_{n}^{2}-s_{n} q_{n}^{2} / g_{n}\right) /\left(\left(K+2 a_{0}\right) q_{n}^{2} / g_{n}-s_{n}\right)$.

Differentiating (B1) a second time with respect to $\alpha_{n}$ and substituting the point $\tilde{\alpha}_{n}=\tilde{\gamma}_{n}$ leads to:

$$
\left.\frac{\partial^{2} \mathcal{L}\left(\boldsymbol{\alpha}, a_{0}, b_{0}\right)}{\partial \alpha_{n}^{2}}\right|_{\widetilde{\alpha}_{n}=\widetilde{\gamma}_{n}}=\frac{-\widetilde{\alpha}_{n}^{-2}\left(s_{n}^{2}-s_{n} q_{n}^{2} / g_{n}\right)}{2\left(\widetilde{\alpha}_{n}+s_{n}\right)\left(\widetilde{\alpha}_{n}+s_{n}-q_{n}^{2} / g_{n}\right)}
$$

Since $s_{n}=\mathbf{\Theta}_{n}^{T} \mathbf{B}_{-n}^{-1} \boldsymbol{\Theta}_{n}$ and $g_{n}=\mathbf{y}^{T} \mathbf{B}_{-n}^{-1} \mathbf{y}+2 b_{0}$ are positive, we should have $s_{n}-q_{n}^{2} / g_{n}>0$ and $\left(K+2 a_{0}\right) q_{n}^{2} / g_{n}-s_{n}>0$ to make $\tilde{\gamma}_{n}>0$. Therefore, $\frac{\partial^{2} \mathcal{L}\left(\boldsymbol{\alpha}, a_{0}, b_{0}\right)}{\partial \alpha_{n}^{2}}$ is always negative at the point $\tilde{\alpha}_{n}=\tilde{\gamma}_{n}$ so $\tilde{\alpha}_{n}=\tilde{\gamma}_{n}$ gives the maximum of the log evidence when $\tilde{\gamma}_{n}>0$. When $\tilde{\gamma}_{n} \leq 0, \tilde{\alpha}_{n}=\infty$ gives the maximum.

In the implementation for updating hyper-parameter $\alpha_{n}$ for Algorithm BCS-IPE, the quantities $\mathbf{C}, q_{m}, s_{m}$, and $g_{m}$ for all terms $(m=1, \ldots, N)$ are needed in each iteration. Efficient updating formulae are presented here following [31]. Updated quantities are denoted by a tilde (e.g. $\tilde{\alpha}_{n}$ ).

In the updating formulae, $g_{m}$ is computed from:

$$
\begin{gathered}
g_{m}=G_{m}+Q_{m}^{2} /\left(\alpha_{m}-\mathcal{S}_{m}\right) \\
G_{m}=\mathbf{y}^{T} \mathbf{B}^{-1} \mathbf{y}+2 b_{0}=\mathbf{y}^{T} \mathbf{y}-\mathbf{y}^{T} \boldsymbol{\Theta} \boldsymbol{\Theta}^{T} \mathbf{y}+2 b_{0}
\end{gathered}
$$

The other quantities $\mathcal{S}_{m}, Q_{m}, s_{m}$, and $q_{m}$ can be found in (A3-A6).

(1) If $\tilde{\gamma}_{n}>0$ and $\alpha_{n}=\infty$, add vector $\boldsymbol{\Theta}_{n}$ and update $\alpha_{n}$, where:

$$
\Delta \mathcal{L}=\frac{1}{2} \log \frac{\alpha_{n}}{\alpha_{n}+s_{n}}-\frac{1}{2}\left(K+2 a_{0}\right) \log \left(1-\frac{q_{n}^{2} / g_{n}}{\alpha_{n}+s_{n}}\right)
$$




$$
\begin{gathered}
\tilde{\mathbf{C}}=\left[\begin{array}{cc}
\mathbf{C}+\mathbf{C}_{n n} \mathbf{C} \boldsymbol{\Theta}^{T} \boldsymbol{\Theta}_{n} \boldsymbol{\Theta}_{n}^{T} \boldsymbol{\Theta C} & -\mathbf{C}_{n n} \mathbf{C} \boldsymbol{\Theta}^{T} \boldsymbol{\Theta}_{n} \\
-\mathbf{C}_{n n}\left(\mathbf{C} \boldsymbol{\Theta}^{T} \boldsymbol{\Theta}_{n}\right)^{T} & \mathbf{C}_{n n}
\end{array}\right] \\
\widetilde{\boldsymbol{\mu}}=\left[\begin{array}{c}
\boldsymbol{\mu}-\mu_{n} \mathbf{C} \boldsymbol{\Theta}^{T} \boldsymbol{\Theta}_{n} \\
\mu_{n}
\end{array}\right]
\end{gathered}
$$

where

$$
\mathbf{C}_{n n}=\left(\alpha_{n}+\mathcal{S}_{n}\right)^{-1}, \boldsymbol{\mu}_{n}=\mathbf{C}_{n n} Q_{n}
$$

For each term $m=1, \ldots, N$ :

$$
\begin{gathered}
\tilde{\mathcal{S}}_{m}=\mathcal{S}_{m}-\mathbf{C}_{n n}\left(\boldsymbol{\Theta}_{m}^{T} \xi_{n}\right)^{2} \\
\tilde{\mathcal{Q}}_{m}=Q_{m}-\mu_{n}\left(\boldsymbol{\Theta}_{m}^{T} \xi_{n}\right) \\
\tilde{G}_{m}=G_{m}-\mathbf{C}_{n n}\left(\mathbf{y}^{T} \xi_{n}\right)^{2}
\end{gathered}
$$

where we define $\xi_{n}=\boldsymbol{\Theta}_{n}-\boldsymbol{\Theta} \mathbf{C} \boldsymbol{\Theta}^{T} \boldsymbol{\Theta}_{n}$.

(2) If $\tilde{\gamma}_{n}>0$ and $\alpha_{n}<\infty$, update $\alpha_{n}$ :

$$
\begin{gathered}
\Delta \mathcal{L}=\frac{1}{2}\left(K+2 a_{0}-1\right) \log \left[1+\mathcal{S}_{n}\left(\tilde{\alpha}_{n}^{-1}-\alpha_{n}^{-1}\right)\right]+\frac{1}{2}\left(K+2 a_{0}\right) \log \frac{\left[\left(\alpha_{n}+s_{n}\right) g_{n}-q_{n}^{2}\right] \tilde{\alpha}_{n}}{\left[\left(\widetilde{\alpha}_{n}+s_{n}\right) g_{n}-q_{n}^{2}\right] \alpha_{n}} \\
\tilde{\mathbf{C}}=\mathbf{C}-\gamma_{j} \mathbf{C}_{j} \mathbf{C}_{j}^{T} \\
\widetilde{\boldsymbol{\mu}}=\boldsymbol{\mu}-\gamma_{j} \mu_{j} \mathbf{C}_{j}
\end{gathered}
$$

where we define $\gamma_{j}=\left(\mathbf{C}_{j j}+\left(\tilde{\alpha}_{n}-\alpha_{n}\right)^{-1}\right)^{-1}, j$ denotes the index within the current basis that corresponds to the single term $n$ to be updated, and $\mathbf{C}_{j}$ is the $j$ th column of $\mathbf{C}$. For each term $m=1, \ldots, N$ :

$$
\begin{gathered}
\tilde{\mathcal{S}}_{m}=\mathcal{S}_{m}+\gamma_{j}\left(\mathbf{C}_{j}^{T} \boldsymbol{\Theta}^{T} \boldsymbol{\Theta}_{m}\right)^{2} \\
\tilde{\mathcal{Q}}_{m}=Q_{m}+\gamma_{j} \mu_{j}\left(\mathbf{C}_{j}^{T} \boldsymbol{\Theta}^{T} \boldsymbol{\Theta}_{m}\right) \\
\tilde{G}_{m}=G_{m}+\gamma_{j}\left(\mathbf{C}_{j}^{T} \boldsymbol{\Theta}^{T} \boldsymbol{\Theta}_{m}\right)^{2}
\end{gathered}
$$

(3) If $\tilde{\gamma}_{n} \leq 0$ and $\alpha_{n}<\infty$, delete vector $\boldsymbol{\Theta}_{n}$ :

$$
\begin{aligned}
\Delta \mathcal{L}=-\frac{1}{2}\left(K+2 a_{0}\right) \log \left(1+\frac{2_{n}^{2} / G_{n}}{\alpha_{n}-\delta_{n}}\right)-\frac{1}{2} \log \left(1-\delta_{n} \alpha_{n}^{-1}\right) \\
\tilde{\mathbf{C}}=\mathbf{C}-\frac{\mathbf{1}}{\mathbf{C}_{j j}} \mathbf{C}_{j} \mathbf{C}_{j}^{T} \\
\tilde{\boldsymbol{\mu}}=\boldsymbol{\mu}-\frac{\mu_{j}}{\mathbf{C}_{j j}} \mathbf{C}_{j}
\end{aligned}
$$


Following updates (B18) and (B19), the appropriate row and column are removed from $\tilde{\mathbf{C}}$ and $\widetilde{\boldsymbol{\mu}}$. For each term $m=1, \ldots, N$ :

$$
\begin{aligned}
& \tilde{\delta}_{m}=\mathcal{S}_{m}+\frac{1}{\mathbf{C}_{j j}}\left(\mathbf{C}_{j}^{T} \boldsymbol{\Theta}^{T} \boldsymbol{\Theta}_{m}\right)^{2} \\
& \tilde{Q}_{m}=Q_{m}+\frac{\mu_{j}}{\mathbf{C}_{j j}}\left(\mathbf{C}_{j}^{T} \boldsymbol{\Theta}^{T} \boldsymbol{\Theta}_{m}\right) \\
& \tilde{G}_{m}=G_{m}+\frac{1}{\mathbf{C}_{j j}}\left(\mathbf{C}_{j}^{T} \boldsymbol{\Theta}^{T} \mathbf{y}\right)^{2}
\end{aligned}
$$

\section{Appendix C: Robustness analysis of Algorithms BCS-MPE and BCS-IPE for signal reconstruction}

It was demonstrated in [18] that the $\log$ evidence function $\mathcal{L}(\boldsymbol{\alpha}, \beta)=\log p(\mathbf{y} \mid \boldsymbol{\alpha}, \beta)$ in (13) can be represented as the difference between the average data-fit term and model complexity (more sparseness corresponds to less model complexity) of the signal model $\mathcal{M}(\boldsymbol{\alpha}, \beta)$. Note that in this study, we make the assumption that the correctly reconstructed signal model corresponds to the global maximum of the evidence. This assumption is consistent with our observations unless the number of compressed measurements $K$ is too small so that there is no chance to recover the original signal correctly.

To investigate the signal reconstruction robustness, , we run the BCS-MPE algorithm using the compressed measurements $\mathbf{y}$ which are obtained by projecting from highly and approximately sparse signals, respectively, and present the results of the calculated log evidences for optimal and several selected suboptimal reconstructed signal models. In each test, the reconstructed signal model that has a tolerably small reconstruction error is regarded as an optimal reconstructed signal model and is denoted by SM 1. We also run the BCS-MPE algorithm for each of three values of $\beta: \exp (2) \times \hat{\beta}, \exp (-2) \times \hat{\beta}$ and $\exp (-4) \times \hat{\beta}$, to get the suboptimal reconstructed signal models SM 2, SM 3 and SM 4, respectively, which have large reconstruction errors. For each signal model, the corresponding log evidences for different $\beta^{\prime} s$ which vary around $\hat{\beta}$ are estimated using (13) by fixing their associated MAP estimate $\widehat{\boldsymbol{\alpha}}$ (or $\breve{\boldsymbol{\alpha}}$, if SM 2, SM3 and SM4) and the results are presented in Figure C1 (a)(i) and (b)(i). When the original signal model is highly sparse (T=5) (Figure C1 (a)(i)), it is seen that the suboptimal reconstructed signal model SM 2 with a larger number of nonzero terms can have larger log evidence for larger values of $\beta$ than the optimal reconstructed signal model SM 1 and so the optimization can be trapped in the local maximum when $\beta=\exp (2) \times \hat{\beta}$. However, for signal models SM 3 and SM 4 which are obtained by fixing $\beta$ smaller than $\hat{\beta}$, the calculated log evidences are always smaller than those of SM 1 . This is due to the fact that the optimal signal model SM 1 is both sufficiently sparse and fits the measurement vector 
well, representing a best balance between data fitting and model complexity in this situation. Therefore, the robustness problem can be alleviated by initializing the optimization for the MAP values in Algorithm BCS-MPE with a very small prediction error precision $\tilde{\beta}$.

For results of approximately sparse signals in Figure $\mathrm{C} 1(\mathrm{~b})(\mathrm{i})$, we observe that the concern of the poor robustness in Algorithm BCS-MPE is even stronger: there are some suboptimal signal models (SM 3 and SM 4) having larger evidence for values of the prediction error precision $\beta$ smaller than $\hat{\beta}$. The reason behind this phenomenon is that optimal signal model SM 1 can fit $\mathbf{y}$ well with small error but the signal model is non-sparse and so its relative entropy is large; on the other hand, the signal models SM 3 and SM 4 are relatively sparse but they cannot fit the measurement vector $\mathbf{y}$ well (data-fit measure is small). The rates of change of the data-fitting and model complexity terms with respect to different $\beta^{\prime} s$ are distinct, which leads to the consequence that the optimal balance between data-fitting and model complexity, and so the maximum peak of $\mathcal{L}(\boldsymbol{\alpha}, \beta)$, is very sensitive to the selection of $\beta$.

In Figure $\mathrm{C} 1$ (a)(ii) and (b)(ii), we employ (25) instead of (13) to calculate log evidence $\mathcal{L}\left(\boldsymbol{\alpha}, b_{0}\right)=$ $\log p\left(\mathbf{y} \mid \boldsymbol{\alpha}, b_{0}\right)$ by using the same MAP estimates $\widehat{\boldsymbol{\alpha}}$ (or $\breve{\boldsymbol{\alpha}}$, if SM 2, SM3 and SM4) for each signal model as in Figure $\mathrm{C} 1(\mathrm{a})(\mathrm{i})$ and (b)(i). The values of $b_{0}$ in Figure C1 (a)(ii) and (b)(ii) are selected simply as the reciprocals of the values of $\beta$ in Figure $\mathrm{C} 1(\mathrm{a})(\mathrm{i})$ and (b)(i), respectively. The comparison results clearly show that considering the uncertainty of $\beta$ leads to a pleasing consequence that the maximum peak of $\mathcal{L}\left(\boldsymbol{\alpha}, b_{0}\right)$ is robust to the selection of the rate parameter $b_{0}$ for both highly and approximately sparse signals, since $b_{0}$ only affects the probability distribution for $\beta$. Therefore, the successive relaxation strategy in the BCS-IPE algorithm which updates $\boldsymbol{\alpha}$ in a iterative fashion for each fixed $b_{0}$ should be robust to find the global maximum of the evidence function.

\section{REFERENCE}

[1]. Candes EJ, Romberg J, Tao T. Robust uncertainty principles: exact signal reconstruction from highly incomplete frequency information. IEEE Trans. Inform Theory 2006; 52(2): 489-509.

[2]. Donoho D. Compressed sensing. IEEE Trans. Inf. Theory 2006; 52(4): 1289-1306.

[3]. Candes EJ, Wakin M. An introduction to compressive sampling. IEEE Signal Proc. Mag. 2008; 25(2): 21 30. 
[4]. Chen SS, Donoho DL, Saunders MA. Atomic decomposition by basis pursuit. SIAM J. Sci. Stat. Comp. 1999; 20(1): 33-61.

[5]. Figueiredo MAT, Nowak RD, Wright SJ. Gradient projection for sparse reconstruction: Application to compressed sensing and other inverse problems. IEEE J. Sel. Topics Signal Process. 2007; 1(4): 586-597

[6]. Tropp JA, Gilbert AC. Signal recovery from random measurements via orthogonal matching pursuit. IEEE Trans. Inf. Theory 2007;53(12):4655-4666.

[7]. Needell D, Tropp JA. CoSaMP: Iterative signal recovery from incomplete and inaccurate samples. Appl. Comput. Harmon. Anal 2009; 26(3):301-321

[8]. Blumensath T, Davies M. Iterative hard thresholding for compressed sensing. Appl. Comput. Harmon. Anal. 2009; 27(3):265-274

[9]. Blumensath T. Accelerated iterative hard thresholding. Signal Proc. 2012; 92(3): 752-756

[10]. Chartrand R. Exact reconstruction of sparse signals via nonconvex minimization. IEEE Signal Proc.Let. 2007; 14(10): 707-710.

[11]. Chartrand R, Yin W. Iteratively reweighted algorithms for compressive sensing. In: Proceedings of ICASSP, 2008; p.3869- 3872

[12]. Ji S, Xue Y, Carin L. Bayesian compressive sensing. IEEE Trans. Sig. Process. 2008; 56(6):2346 -2356.

[13]. Babacan S, Molina R, Katsaggelos A. Bayesian compressive sensing using Laplace priors. IEEE Trans Sig. Process. 2010;19(1):53-64

[14]. Tipping ME. Sparse Bayesian learning and the relevance vector machine. J. Mach. Learn Res 2001;1: 211-244.

[15]. Tipping ME, Faul AC. Fast marginal likelihood maximization for sparse Bayesian models. In: Proceedings of 9th International Workshop on Artificial Intelligence and Statistics, Key West, FL, 2003.

[16]. Wipf D, Rao B. Sparse Bayesian learning for basis selection. IEEE Trans. Sig. Process. 2004; 52(8):21532164.

[17]. Wipf D, Rao B. Comparing the effects of different weight distributions on finding sparse representations. Adv. Neural Inf. Process. Syst. 2006;18:1521-1528.

[18]. Huang Y, Beck JL, Wu S, Li H. Robust Bayesian compressive sensing for signals in structural health monitoring. Comput.Aided Civ. Inf. 2014; 29(3):160-179. 
[19]. Barbier J, Krzakala F, Mézard M, Zdeborová L. Compressed sensing of approximately-sparse signals: phase transitions and optimal reconstruction. In: Proceedings of 50th Annual Allerton Conference on Communication, Control, and Computing, Monticello, IL, 2012; p. 800-807.

[20]. Gilbert AC, Li Y, Porat E, Strauss MJ. Approximate sparse recovery: optimizing time and measurements. SIAM J. Comput. 2009; 41(2): 436-453.

[21]. Stojnic M, Xu WY, Hassibi B. Compressed sensing of approximately sparse signals. In: Proceedings of ISIT, Toronto, ON, 2008; 2182- 2186.

[22]. Huang Y, Beck JL, Wu S, Li H. Robust diagnostics for Bayesian compressive sensing with applications to structural health monitoring, In: Proceedings of SPIE Smart Structures and Materials + Nondestructive Evaluation and Health Monitoring, San Diego, USA, 2011.

[23]. Peckens CA, Lynch JP. Utilizing the cochlea as a bio-inspired compressive sensing technique, Smart Mater. Struct. 2013; 10:105027.

[24]. Bao YQ, Li H, Sun XD, Ou JP. Compressive sampling based data loss recovery for wireless sensor networks used in civil structural health monitoring. Struct.Health Monit. 2013;12(1):78-95.

[25]. Meyer J, Bischoff RB, Feltrin G. Wireless sensor network for long-term structural health monitoring. Smart Struct Syst 2006; 6(3): 263-275.

[26]. Yang Y, Nagarajaiah S. Output-only modal identification by compressed sensing: non-uniform low-rate random sampling, Mech. Syst. Signal Process. 2015; 56: 15-34.

[27]. Mascareñas D., Cattaneo A., Theiler, J., Farrar, C. Compressed sensing techniques for detecting damage in structures, Structural Health Monitoring 2013; 12(4):325-338.

[28]. Huang Y, Beck JL, Hierarchical sparse Bayesian learning for structural health monitoring with incomplete modal data, International Journal for Uncertainty Quantification 2015; 5(2):139-169.

[29]. Beck JL. Bayesian system identification based on probability logic. Struct. Control. Health Monit 2010; 17 (7): 825-847.

[30]. Beck JL, Katafygiotis LS. Updating models and their uncertainties I: Bayesian statistical framework. J. Eng. Mech. 1998; 124:455-461.

[31].Ji S. Dunson, D. and Carin, L. Multi-task compressive sensing. IEEE Trans. Sig. Process. 2009; 57(1): 92-106.

[32]. Duke University, Bayesian Compressive Sensing. 
http://people.ee.duke.edu/ lcarin/BCS.html

[33]. Northwestern University, Image and Video Processing Laboratory, Software.

http://ivpl.eecs.northwestern.edu/software

[34].11-Magic.

http://statweb.stanford.edu/ candes/11magic/

[35].Instituto de Telecomunicações, GPSR.

http://www.lx.it.pt/ mtf/GPSR/

[36]. Thomas Blumensath, software.

http://www.personal.soton.ac.uk/tb1m08/sparsify/sparsify.html

[37]. Ou JP, Li H. Structural Health Monitoring in mainland China: Review and Future Trends. Structural Health Monitoring 2010; 9:219-231

[38].Li SL, Li H, Liu Y, Lan CM, Zhou WS, Ou JP. SMC structural health monitoring benchmark problem using monitored data from an actual cable-stayed bridge. Struct.Control. Health Monit. 2014; 21(2):156-172. 
(i)

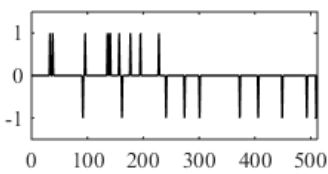

$\mathrm{RE}=0.0189$

(ii)

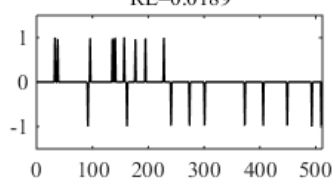

(iii)

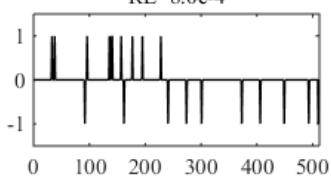

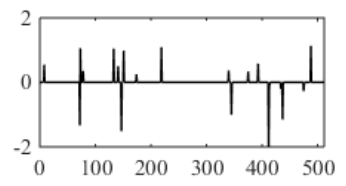

$\mathrm{RE}=0.0396$

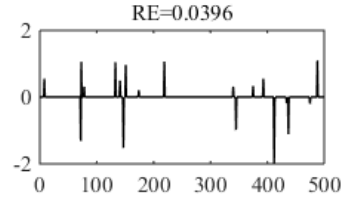

$\mathrm{RE}=9.3 \mathrm{e}-3$

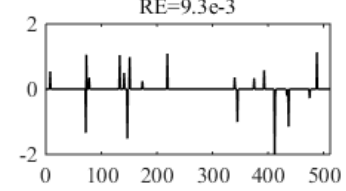

(i)

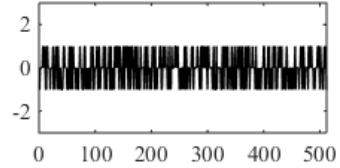

$\mathrm{RE}=0.6928$

(ii)

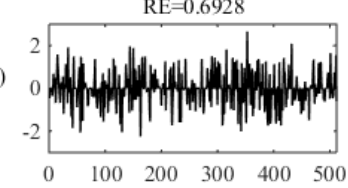

$\mathrm{RE}=5.4 \mathrm{e}-5$

(iii)

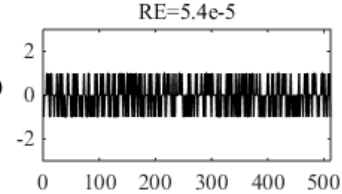

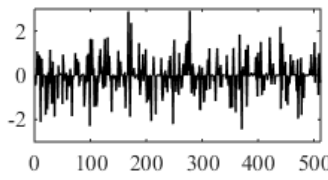

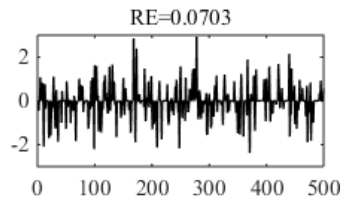

$\mathrm{RE}=4.8 \mathrm{e}-4$

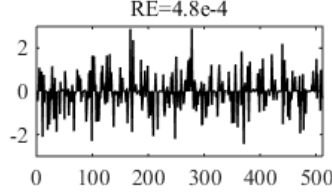

(a)

(b)

(d)

Figure 1. (i) Original signal and (ii) BCS-MPE and (iii) BCS-IPE mean reconstructed signals of length $N=512$ for four signals: (a) Uniform spikes, $T=20$; (b) Non-uniform spikes, $T=20$; (c) Uniform spikes, $T=250$; (d) Non-uniform spikes, $T=250$. The number of measurements $K$ is selected as 90, 60, 470 and 380 for the four signals in (a), (b), (c) and (d), respectively. RE denotes the reconstruction error defined in the text. 


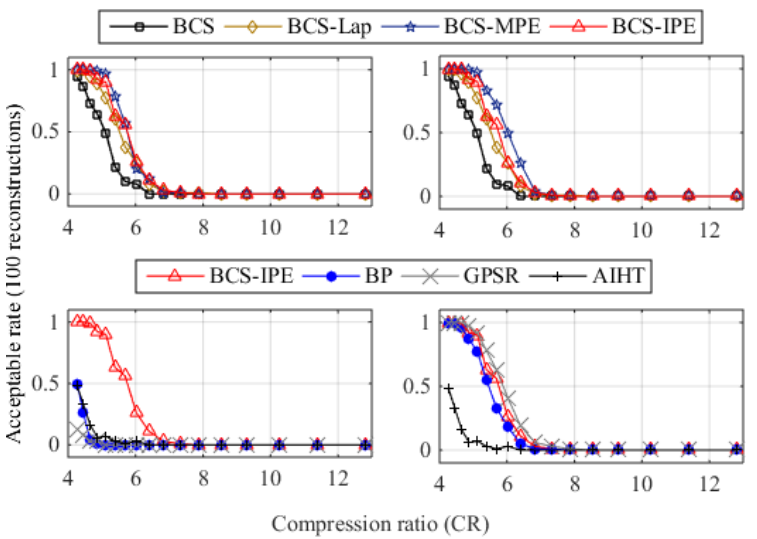

(i)

(i)

(a)

(b) Non-uniform spikes ( $\mathrm{T}=20)$

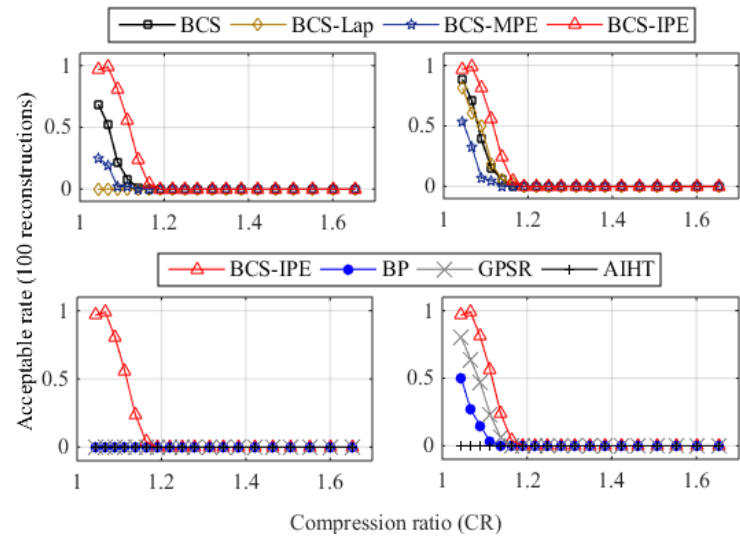

(i)

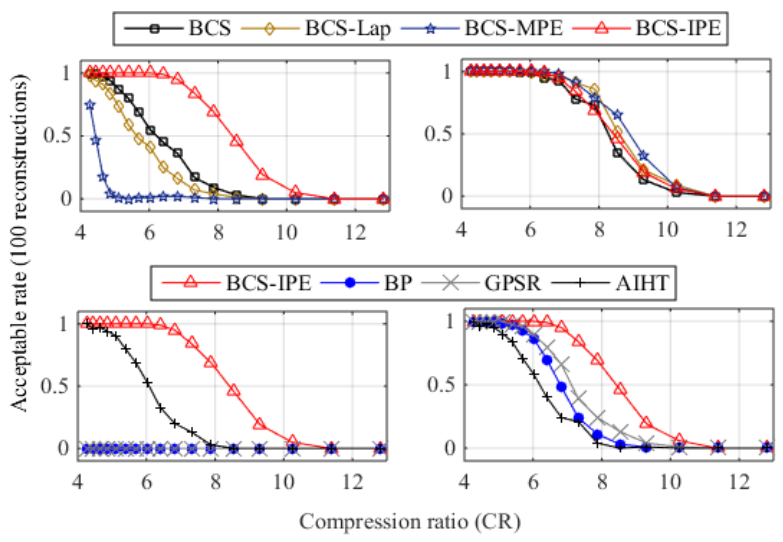

(ii)

(ii)

spikes

$(\mathrm{T}=20)$

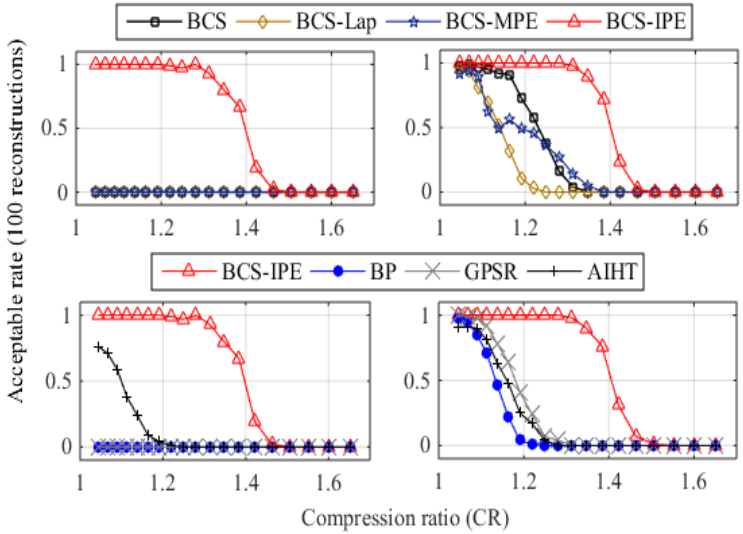

(ii)

(i)

(ii)

(d) Non-uniform spikes ( $\mathrm{T}=250)$

Figure 2. Relation of compression ratio and the rate of acceptable reconstruction error for two different thresholds of (i) 0.02 ; (ii) 0.2, by running seven CS reconstruction algorithms. 
(i)

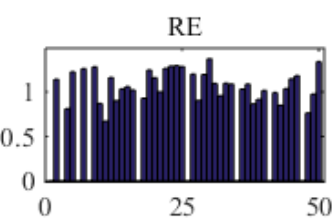

(ii)

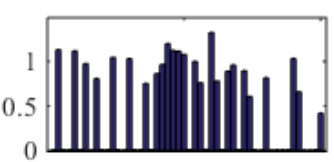

(iii) 0.5

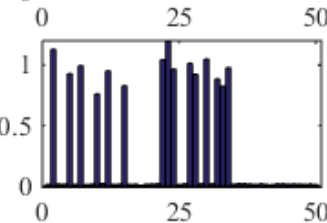

(iv)
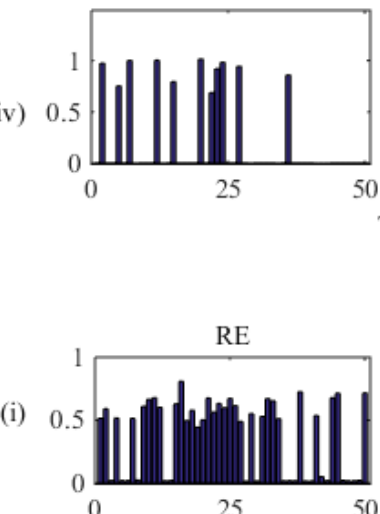

(ii)

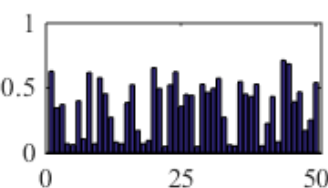

(iii)

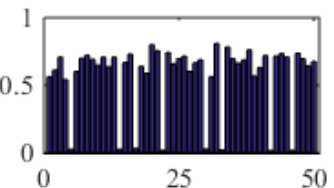

(iv) 0

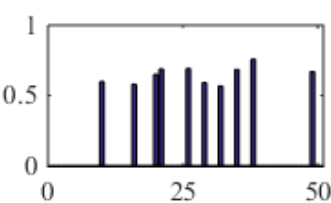

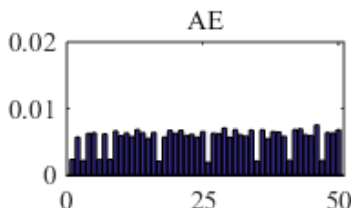

0

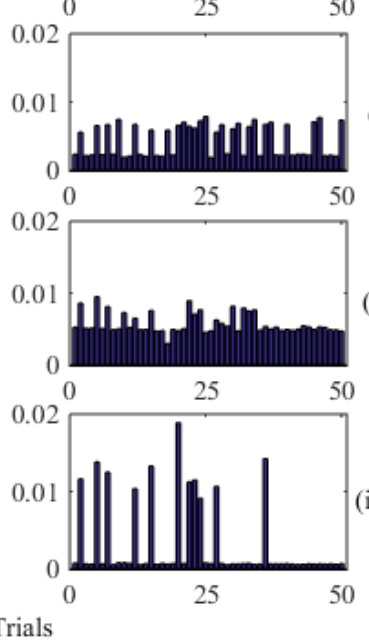

(a)
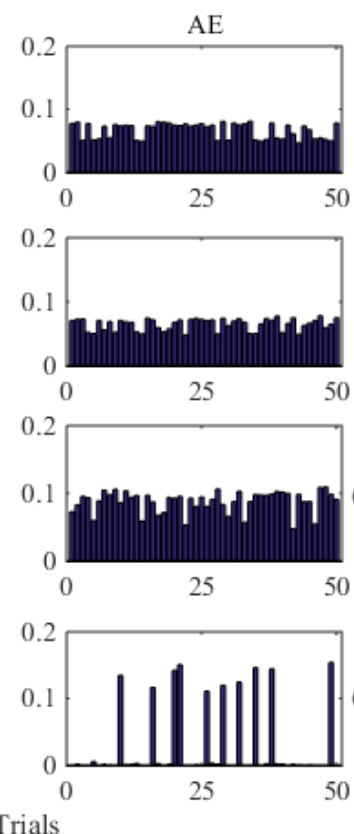

(c)

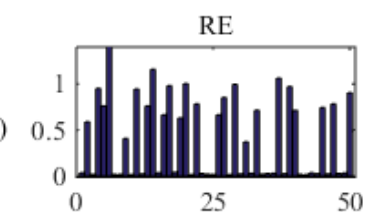

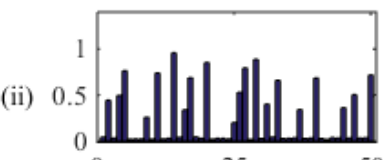
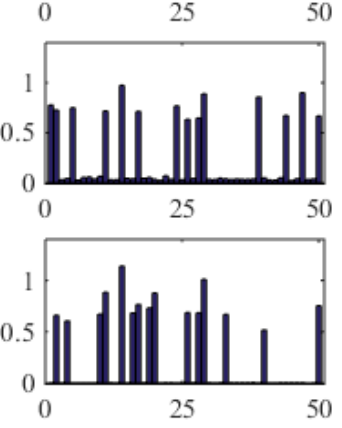

RE

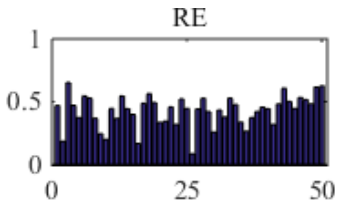

(ii)

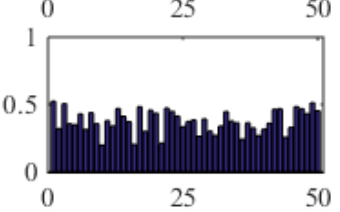

(iii)

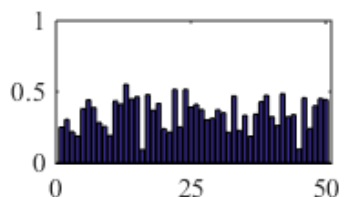

(iv) 0.5

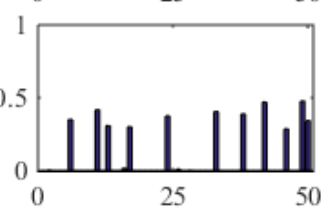

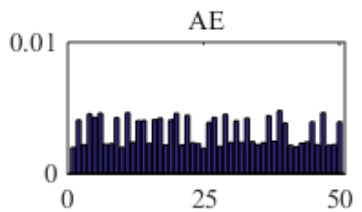
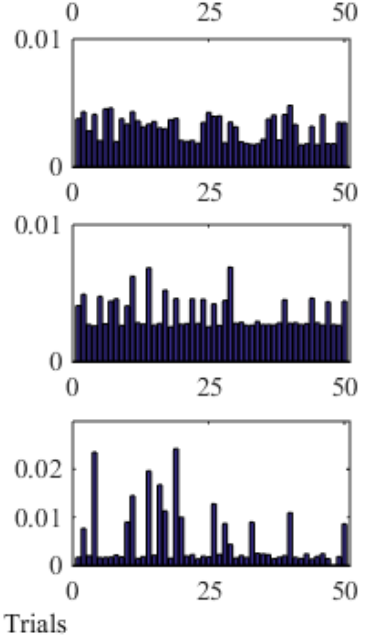

(b)
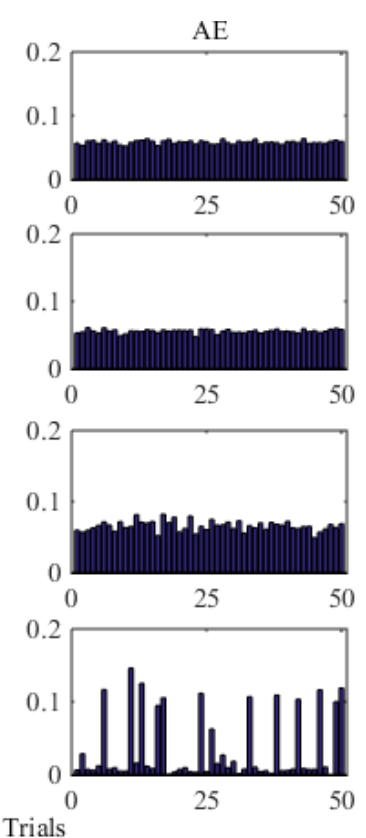

(d) 
Figure 3. Reconstruction errors (RE) and the corresponding average error bars (AE) for 50 runs for each of four signals: (a) Uniform spikes, $T=20$; (b) Non-uniform spikes, $T=20$; (c) Uniform spikes, $T=250$; (d) Non-uniform spikes, $T=250$, and using four BCS algorithms: (i) BCS; (ii) BCS-Lap; (iii) BCS-MPE and (iv) BCS-IPE.
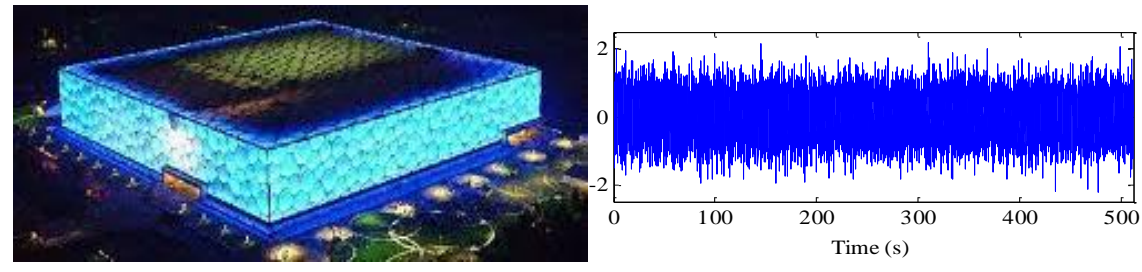

Figure 4. Case 1: Beijing National Aquatics Center and its investigated acceleration response. 

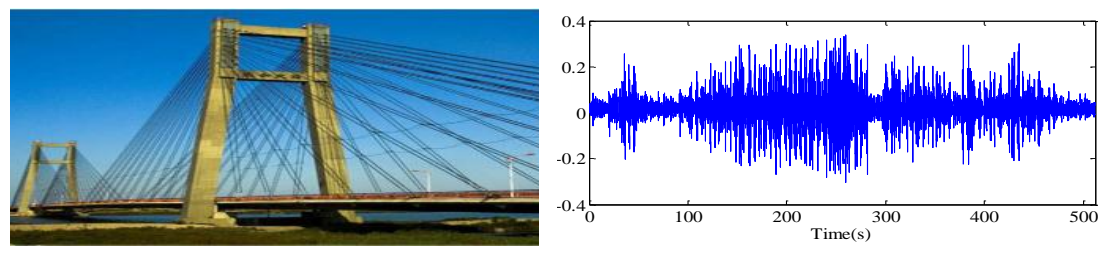

Figure 5. Case 2: Tianjin Yonghe Bridge and its investigated acceleration response. 


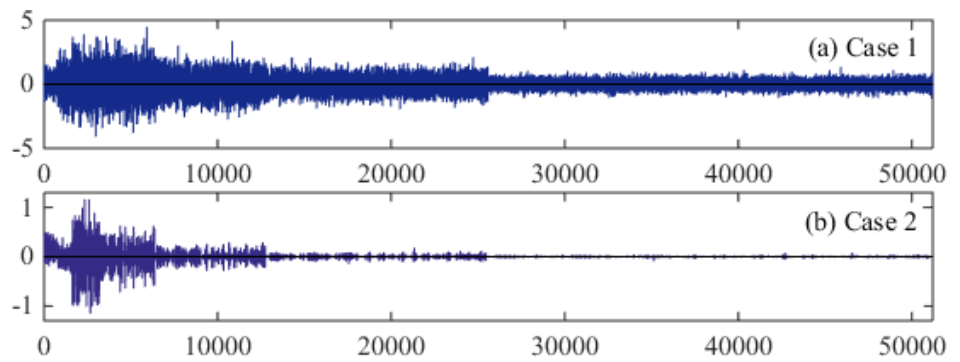

Figure 6 Wavelet coefficients of the acceleration data for Cases 1-2 using Haar wavelet basis. 

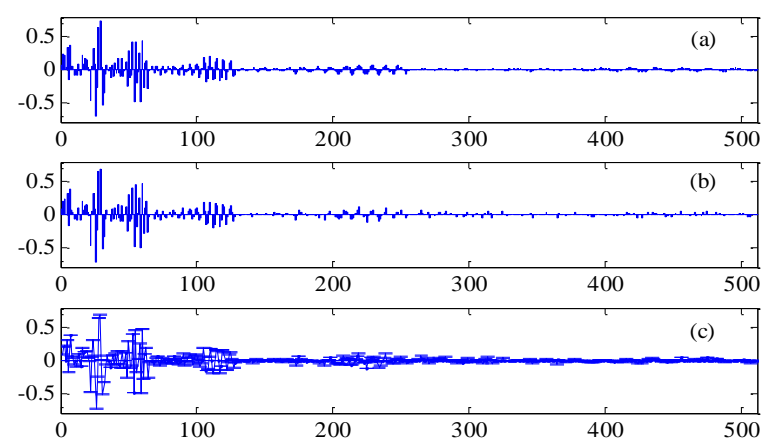

Figure 7. (a) The wavelet coefficients of the first time segment of length $N=512$ of the acceleration signal from the Tianjin Yonghe Bridge (Case 2); Posterior mean (b) and error-bars (c) of reconstructed wavelet coefficients using BCS-IPE with $K=340$ for the compressed data. The closely-spaced upper and lower short horizontal lines for each reconstructed wavelet coefficient delineate the error bars, with the centers of the vertical lines corresponding to the posterior mean. 

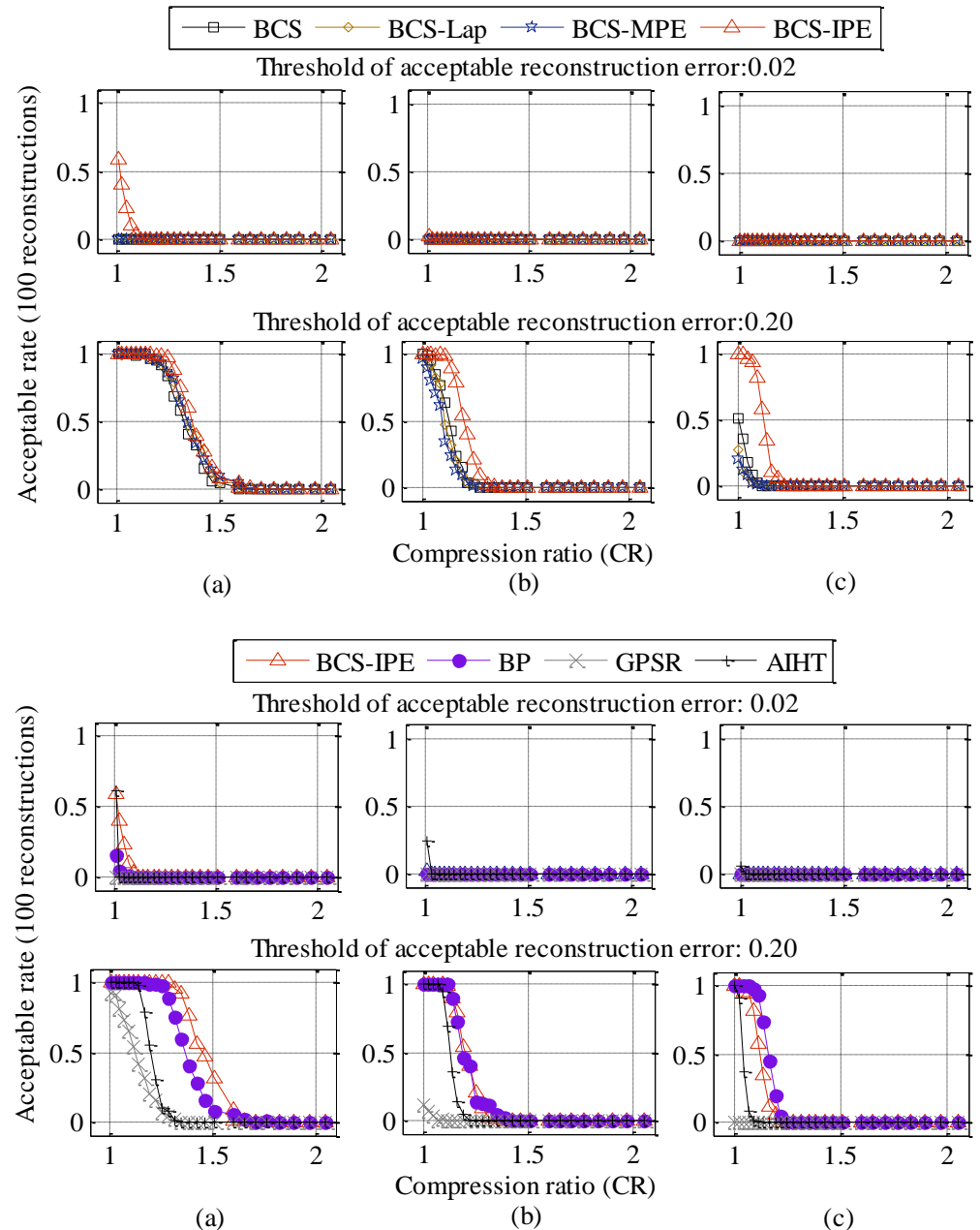

Figure 8: Case 1 (Original signal from Beijing National Aquatics Center): Relation of compression ratio and the rate of acceptable reconstruction error for two different thresholds for the reconstruction errors: (a) 1/16 largest coefficients; (b)

1/4 largest coefficients; and (c) all coefficients of the original wavelet coefficient vector. 


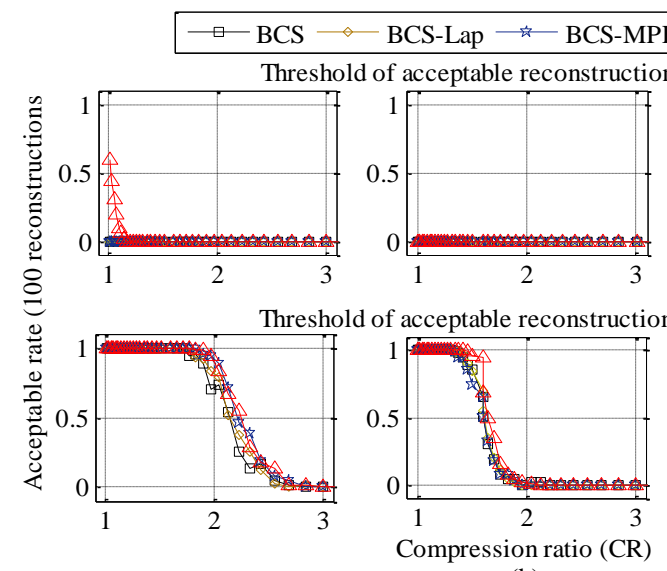

(a)
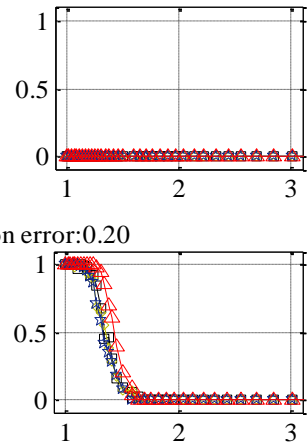

(c)

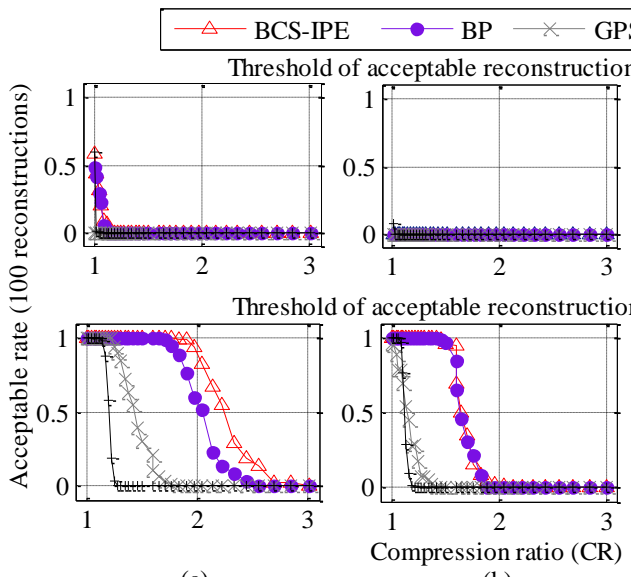

(b)
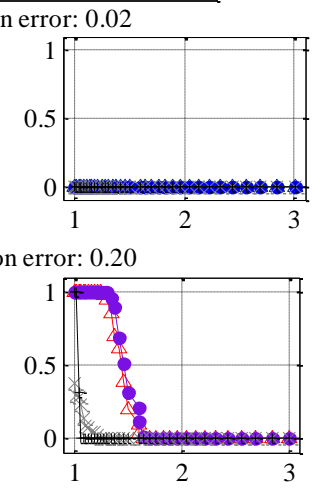

(c)

Figure 9: Case 2 (Original signal from Tianjin Yonghe Bridge): Relation of compression ratio and the rate of acceptable reconstruction error for two different thresholds for the reconstruction errors: (a) 1/16 largest coefficients; (b) $1 / 4$ largest coefficients; and (c) all coefficients of the original wavelet coefficient vector. 


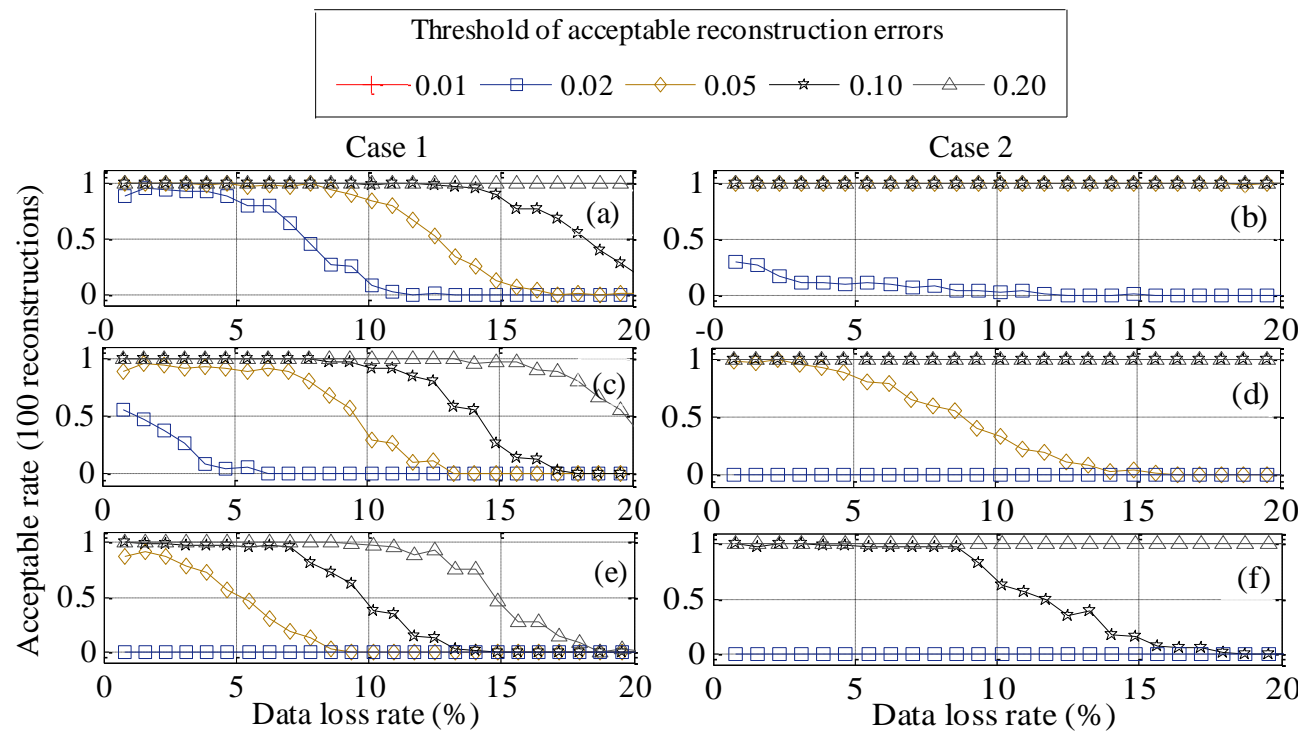

Figure 10: Relation between data loss rate and the rate of acceptable reconstruction error for 5 different thresholds for the reconstruction errors for BCS-IPE of (a),(b): 1/16 largest coefficients, (c),(d): 1/4 largest coefficients, and (e),(f): all coefficients of the original wavelet coefficient vector for Case 1: (a), (c), (e) (Beijing National Aquatics Center), and Case 2: (b), (d), (f) (Tianjin Yonghe Bridge) . 


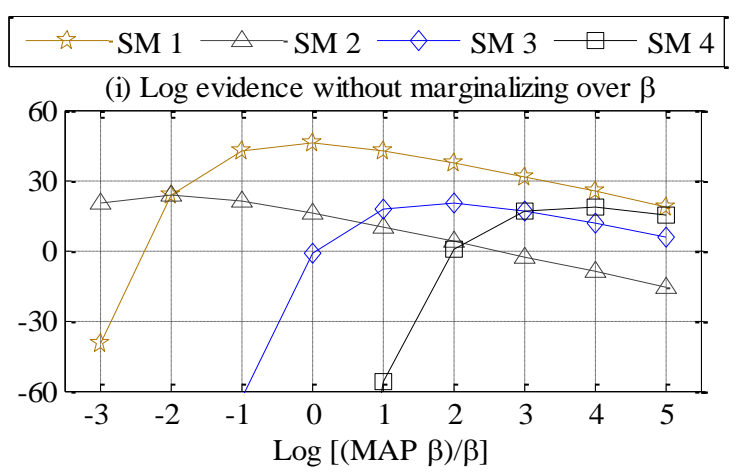

(ii) Log evidence when marginalizing over $\beta$

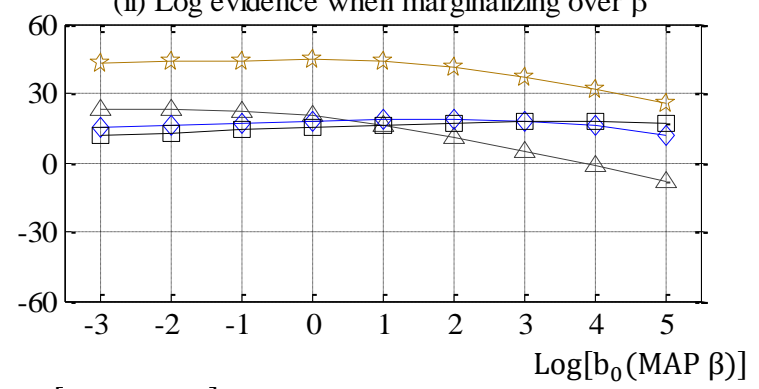

$\log \left[\mathrm{b}_{0}(\mathrm{MAP} \beta)\right]$

(a) $T=5$

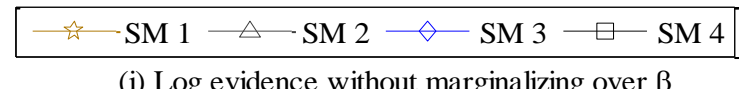

(i) Log evidence without marginalizing over $\beta$

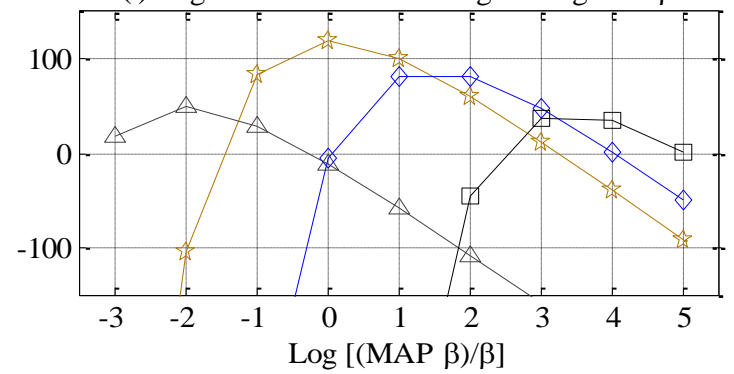

(ii) Log evidence when marginalizing over $\beta$

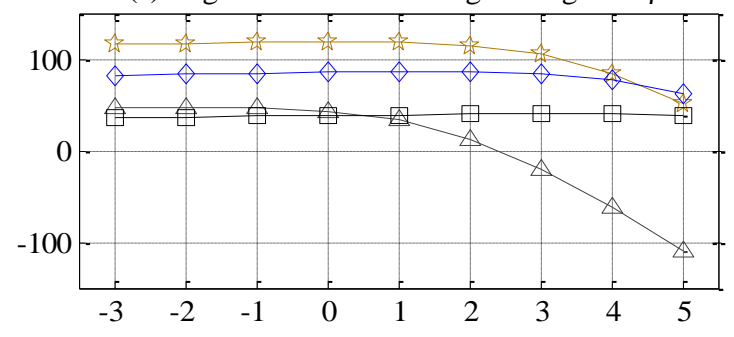

(b) $T=60$

Figure C1. Calculated log evidence for four reconstructed signal models (SMs): SM 1: optimal signal model from a run of BCS-MPE; SM 2: a run of BCS-MPE with $\beta$ fixed at $\exp (-2) \times \hat{\beta}$; SM 3: a run of BCS-MPE with $\beta$ fixed at $\exp (2) \times$ $\hat{\beta}$; and SM 4: a run of BCS-MPE with $\beta$ fixed at $\exp (4) \times \hat{\beta}$. By fixing the MAP estimates $\widehat{\alpha}$ for each signal model, the $\log$ evidence is calculated by: (i) using (13) for different choices of $\beta$; (ii) using (25) for different choices of $b_{0}$. The original signal is non-uniform spikes of length $N=126$ and with number of nonzero terms: (a) $T=5$; (b) $T=60$. For (a), the reconstruction errors for signal models SM 1-4 are 7.29e-6, 0.4354, 0.4231 and 0.3921 , respectively; the number of nonzero terms for signal models SM 1-4 is 5, 12, 11 and 10, respectively, while for (b), the reconstruction errors for signal models SM 1-4 are 9.58e-004, 0.2533, 0.2733 and 0.3124 , respectively; the number of nonzero terms for SM $1-4$ is $60,95,56$, and 48 , respectively. 
Table 1. Average running time (s) for four signals using different algorithms

\begin{tabular}{ccccc}
\hline Algorithms & $\begin{array}{c}\text { Uniform spikes } \\
\mathrm{T}=20\end{array}$ & $\begin{array}{c}\text { Non-uniform spikes } \\
\mathrm{T}=20\end{array}$ & $\begin{array}{c}\text { Uniform spikes } \\
\mathrm{T}=250\end{array}$ & $\begin{array}{c}\text { Non-uniform } \\
\text { spikes } \mathrm{T}=250\end{array}$ \\
\hline BCS & 0.09 & 0.08 & 2.26 & 1.75 \\
BCS-Lap & 0.35 & 0.24 & 2.93 & 2.59 \\
BCS-MPE & 0.80 & 0.36 & 16.23 & 14.01 \\
BCS-IPE. & 1.05 & 0.76 & 21.85 & 16.86 \\
BP & 0.69 & 1.87 & 3.14 & 3.83 \\
GPSR & 0.57 & 0.56 & 1.04 & 0.52 \\
AIHT & 0.01 & 0.03 & 0.10 & 0.15 \\
\hline
\end{tabular}

